\title{
DIRICHLET PROBLEMS ON RIEMANN SURFACES AND CONFORMAL MAPPINGS
}

\author{
MAKOTO OHTSUKA
}

\author{
Contents \\ Introduction \\ Chapter I. Perron-Brelot's method and the types of Riemann surfaces. \\ 1. Perron-Brelot's method. \\ 2. The types of Riemann surfaces. \\ Chapter II. Existence problems. \\ 1. Existence theorem on $\Re$ with positive boundary. \\ 2. Existence theorem on $\mathfrak{A}$ with null boundary. \\ 3. Variation of preassigned singularities. \\ 4. The simplest harmonic functions on $\mathfrak{\Re}$. \\ Chapter III. Boundary correspondences in conformal mappings. \\ 1. One-to-one correspondence between boundaries. \\ 2. Measure of the image of the accessible boundary points. \\ 3. Mapping of an abstract Riemann surface. \\ 4. Regular points. \\ 5. Ideal boundary components. \\ 6. Parabolic fixed points. \\ 7. Hyperbolic fixed points. \\ 8. Non-fixed singular points. \\ Chapter IV. Dirichlet problems. \\ 1. Problem on an open compact set in $\Re$. \\ 2. Problem on $\mathfrak{A}$ as a covering surface. \\ 3. Applications. \\ 4. Regular points. \\ Chapter V. Conformal mapping of a Riemann surface with a rectifiable \\ boundary. \\ 1. A lemma from the theory of cluster sets. \\ 2. Measure-theoretic lemmas. \\ 3. Rectifiability of boundary of a Riemann surface. \\ 4. Continuity of the mapping function. \\ 5. Correspondence of null sets. \\ 6. Equalities for the length. \\ Bibliography.
}

Received May 2, 1951. 


\section{Introduction}

The object of this paper is an investigation of existence problems and Dirichlet problems on an abstract Riemann surface in the sense of Weyl-Radón or on a covering surface over it,") and of boundary correspondence in the conformal mapping of the surface.

In 1923 O. Perron introduced a new method to Dirichlet problem ${ }^{3)}$ and this was developed by M. Brelot.4) M. H. Heins remarked in 1949 that this method is available also on a compact domain in a Riemann surface, ${ }^{5)}$ and it has been used after him.' In Chapter I, $\S 1$ a brief exposition of this method is given, and in $\S 2$ abstract Riemann surfaces are classified into two types after $R$. Nevanlinna ;) either with null or positive boundary. The types can be decided by Perron-Brelot's method without using any exhaustion, and the separability of any Riemann surface ${ }^{\text {s) }}$ can be deduced from the existence of a harmonic measure. Further some properties of surfaces with null boundary are stated there in preparation for the next chapter.

Chapter II is concerned with existence problems of harmonic functions on Riemann surfaces, which have the singularities given in compact parts. The well-known method using the Dirichlet principle is described in detail in $\mathrm{H}$. Weyl [1]. Along this line he published the method of orthogonal projection" and this method has been used by $\mathrm{K}$. Kodaira to show the existence of functions. ${ }^{10)}$ Also L. Ahlfors strengthened the base of the Dirichlet principle.11 The harmonic functions thus obtained have finite Dirichlet integrals outside the singularities. Another method is alternierendes Verfahren due to H. A. Schwarz and C. Neumann. The latter showed already in 19-century the existence of the harmonic function having a preassigned singularity on a closed covering surface over a Riemann sphere, ${ }^{12}$ and this existence theorem was extended to the case when

\footnotetext{
1) This is a complex 1-dimensional analytic manifold. Cf. H. Weyl [1], T. Radó [1].

2) As to covering surface, see $S$. Stoilow [1].

3) O. Perron [1]. Cf. also R. Remak [1], T. Radó and. F. Riesz [1], C. Carathéodory [3].

4) M. Brelot [2], [4].

5) M. H. Heins [1]. Cf. also L. Ahlfors [2].

6) In M. Parreau [1], for instance.

7) R. Nevanlinna [3].

8) This was first proved in T. Radó [1].

9) H. Weyl [2].

1i) K. Kodaira [1], [2].

11) L. Ahlfors [3], [4].

12) C. Neumann [1]. Cf. also W. F. Osgood [1].
} 
the surface has a null boundary by R. Nevanlinna. ${ }^{13)}$ Quite recently L. Sario applied alternierendes Verfahren more widely ${ }^{14)}$ and $R$. Nevanlinna discussed existence problems from the viewpoint of integral equations. ${ }^{15)}$ When the surface has a null boundary, the solution, which is bounded outside the singularity given in a compact part, has a finite Dirichlet integral there and vice versa. ${ }^{16)}$ But this is not true generally, when the surface has a positive boundary. ${ }^{17}$ In Chapter II the existence problems are discussed by Perron-Brelot's method on a surface with positive boundary, and by a method due to M. H. Heins ${ }^{18)}$ on a surface with null boundary. ${ }^{19}$

Once existence theorems are established, uniformization becomes possible; for instance, by Koebe's original proof..$^{20}$ Chapter III is devoted to the study of boundary correspondences in conformal mappings. In the first half the image on the unit circle $\Gamma:|z|=1$ of accessible boundary points is investigated when the universal covering surface of $\mathfrak{R}$ is mapped conformally onto a unit circular domain $U:|z|<1,21)$ where $\Re$ is a covering surface over an abstract Riemann surface $\mathfrak{R}$. In $R$. Nevanlinna [3] he stated without detailed proof that if the transfinite kernel of $\Re-\Re$ is non-empty on $\Re$ then the measure of the image on $\Gamma$ of the accessible boundary points is $2 \pi$ under the following assumptions: (A) the projection of $\mathfrak{R}$ is compact in $\mathfrak{R}$; (B) $\mathfrak{R}$ covers $\underline{\mathfrak{R}}$ simply, and proposed the question: Is it possible to remove the assumption (B)? An answer to it is given in $\S 2$. Next, the boundary correspondence in the mapping of multiplyconnected plane domains was studied systematically in the first edition of $L$. Bieberbach [1], but some parts were omitted in the second edition. R. Nevanlinna and $O$. Teichmüller ${ }^{22)}$ pointed out the faults in the first edition and corrected some of them, but the part concerning parabolic fixed points remains still indistinct. In the second half of this chapter the correspondence between ideal boundary

\footnotetext{
13) R. Nevanlinna [5].

14) L. Sario [1].

15) R. Nevanlinna [7], [8].

16) This was shown in R. Nevanlinna [6].

17) L. Sario [1] discussed a case in which a solution exists such that it is bounded and has a finite Dirichlet integral at the same time outside the singularity.

18) M. H. Heins [1].

19) I could not refer to the works in ${ }^{14)}$ and ${ }^{15)}$ until my manuscript was almost completed, and have no access to the details of some of them still now.

20) P. Koebe [1].

21) Throughout this paper $\Gamma$ and $U$ denote $|\boldsymbol{z}|=1$ and $|\boldsymbol{z}|<1$ respectively.

22) R. Nevanlinna [1]. O. Teichmüller [1].
} 
components (Randstiicke due to B.v. Kerékjártó ${ }^{23)}$ ) of an abstract Riemann surface and the points on $\Gamma$ are studied by making use of the corrected form of Bieberbach's method.

Chapter IV deals with Dirichlet problems on Riemann surfaces. $\S 1$ is devoted to the continuation of Chap. I, $\S 1$. It is shown there that any continuous boundary function is resolutive, $\left.{ }^{24}\right)$ and that the similar considerations as in euclidean spaces are possible. Next let $\mathfrak{R}$ be a covering surface over an abstract Riemann surface, with relative boundary and satisfying (A) and the following condition: $u(P) \leqq 0$ on $\mathfrak{R}$ whenever $u(P)$ is upper bounded continuous subharmonic such that $\lim _{\mathfrak{I}} u(P) \leqq 0$, where $\mathfrak{I}_{\mathfrak{R}}$ denotes all the accessible boundary points. In $\S \S 2-4$ Dirichlet problem is treated on $\mathfrak{A}$ by making use of the theory of conformal mapping in the special case when the connectivity of $\Re$ is finite. But it is desirable that some resolutive functions can be assured without using the theory of conformal mapping and in general case. The concept of regular and irregular points on the boundary is important in the theory of Dirichlet problem. In the present case, however, I have scarecely any knowledge about them. Thus there are left many questions open in this chapter. Moreover it may be interesting to investigate potential theory on Riemann surfaces further in connexion with the recent researches by $\mathrm{R}$. Bader and M. Parreau, ${ }^{251}$ or to treat Dirichlet problem on an abstract Riemann surface having all the ideal boundary components as its boundary.

Now let $w=f(z)$ be a regular bounded function in $U$, and suppose that it is continuous on $U+T$ and the length of the $w$-image of $T$ is finite. It was proved by Brother Riesz that the null sets on $\Gamma$ and the sets of zero length on the image of $\Gamma$ correspond to each other. ${ }^{26)}$ Given a domain $D$ in the $w$-plane, when does the mapping function of $U$ onto $D$ satisfy the condition stated above? It is surely so when $D$ is bounded by a rectifiable Jordan curve. W. Seidel defined the length of the boundary of any simply-connected plane domain and showed that if the length is finite the mapping function satisfies the required condition.": In Chapter $\mathrm{V}$ such problem is treated for a covering surface $\Re$ over a metric Riemann surface $\sigma$. Under some additional conditions I define the rectifiability of the boundary of $\mathfrak{R}$, and show that the mapping function of $U$ into $\sigma$ has similar properties as above $f(z)$ and that Riesz's theorem holds good also in this case.

23) B. v. Kerékjártó [1]. Cf. also S. Stoïlow [1].

24) For the definition of resolutivity, see Chap. I, $\S 1$.

25) R. Bader [1]. M. Perreau [2]. Cf. also L. Ahlfors [1].

:6) F. and M. Riesz [1]. Cf. also F. Riesz [1], N. Lusin and J. Priwaloff [1], M. Tsuji [1].

$\therefore$ W. Seidel [1], esp. pp. 194-204. 
It seems to me that, in addition to the subjects taken up in this paper, there remain still many, which have ever been discussed only on planes but may be treated on Riemann surfaces with interest.

I wish to express my warmest gratitude to Prof. Noshiro for his perpetual encouragement and valuable remarks during my investigation.

\section{Chapter I. Perron-Brelot's Method and the \\ Types of Riemann Surfaces}

\section{Perron-Brelot's method ${ }^{2 s)}$}

We shall explain Perron-Brelot's method for Dirichlet problem on an abstract Riemann surface $\mathfrak{R}$ in this section.

The following lemma used by M. Brelot in euclidean spaces holds good also on $\mathfrak{R}$ :

LEMMA 1.1. The upper cover (defined by the supremum at each point) of a class of positive harmonic functions in a domain on $\Re$ is continuous subharmonic or equal to the constant $+\infty$.

For an open compact true subset $D$ in $\Re$ and a real function $\varphi$ (admitting $\pm \infty$ ) defined on the boundary $C^{29}$ of $\mathscr{D}$, the lower class $11 \mathfrak{P}$ is defined by all the upper bounded continuous subharmonic functions $\{u(P)\}$ such that $\lim _{P \rightarrow P^{\prime} \in C} u(P)$ $\leqq \varphi\left(P^{\prime}\right)$, adding $-\infty$ to $\mathfrak{l}_{\varphi}^{D}$. Then it follows by Lemma 1.1 that the upper cover $H_{p}^{D}(P)$ of $\mathfrak{u}_{p}^{D}$, which will be called hypo function, is harmonic or equal to the constant $+\infty$ or $-\infty$ in each component of $\mathscr{D}$. Similarly the upper class $\mathfrak{N}: \mathcal{P}$ and its lower cover $\bar{H}_{p}^{D}(P)$, which will be called hyper function, are defined for superharmonic functions and $\bar{H}_{\varphi}^{\mathscr{D}}(P)$ has the similar character as $\underline{H}_{\mathcal{Q}}^{\mathscr{T}}(P)$. Further, $\underline{H}_{\mathscr{P}}(P) \leqq \bar{H}_{\mathscr{p}}(P)$ on $\mathscr{D}$, and if they coincide at a point they are identical in the component containing the point. When $\underline{H}_{\mathcal{P}}(P) \equiv \bar{H}_{\mathcal{P}}^{\mathfrak{D}}(P)$, we shall denote it by $H_{P}(P)$ and call it the general solution, and if, in addition, it is finite, $\varphi$ will be called a resolutive boundary function.

A point $P^{\prime}$ on $C$ will be called regular or irregular if in a local parameter circle the image of $P^{\prime}$ is regular or irregular with respect to the image of $I^{3}$. This definition does not depend upon the local parameter selected there. At a regular point $P^{\prime}$ a barrier ${ }^{31)}$ exists and by it there holds

23) As to the contents of this section, cf. M. Brelot [2].

29) Here, the boundary point is the one in the usual sense; it is the point, in any whose neighborhood both $\mathfrak{D}$ and $\mathfrak{A}-\mathfrak{D}$ enter. It will be called an ordinary boundary point.

30) Dirichlet problem on a plane is supposed to be known.

31) We accept the barrier in a wider sense; it is continuous superharmonic on $\mathfrak{D}$, tends to zero as $P \rightarrow P^{\prime}$, and its lower bound outside every neighborhood of $P^{\prime}$ is positive. 


$$
\varlimsup_{P \rightarrow P^{\prime}} \bar{H}_{\varphi}(P) \leqq \varphi\left(P^{\prime}\right),
$$

if $\varphi$ is upper bounded on $C$ and upper semicontinuous at $P^{\prime}$, and

$$
\lim _{P \rightarrow P^{\prime}} \underset{\varphi}{\mathscr{D}}(P) \supseteqq \varphi\left(P^{\prime}\right),
$$

if $\varphi$ is lower bounded on $C$ and lower semicontinuous at $P^{\prime}$. Consequently if $\varphi$ is bounded on $C$ and continuous at a regular point $P^{\prime}$, there holds

$$
\lim _{P \rightarrow P^{\prime}} H_{\varphi}(P)=\lim _{P \rightarrow P^{\prime}} \bar{H}_{\varphi}^{\Phi}(P)=\varphi\left(P^{\prime}\right) .
$$

If every point of $C$ is regular and $\varphi$ is continuous on $C$, follows $\underline{H}_{\varphi}^{\Phi}(P) \equiv \bar{H}_{\phi}(P)$ and this gives the solution of the Dirichlet problem in the classical sense; for instance, it is so on a domain bounded by Jordan curves.

Near a simple closed Jordan curve $\gamma$ draw another simple closed Jordan curve $\gamma^{\prime}$ and enclose a doubly-connected domain by them. The $\varepsilon$-niveau curve of the solution with the boundary value 0 on $\gamma$ and 1 on $\gamma^{\prime}$ is a simple closed analytic curve. Hence in an exhaustion $\left\{\Re_{n}\right\}$ of $\Re$ we may, and shall, suppose that each $\Re_{n}$ is bounded by a finite number of analytic curves and no component of its complement is compact in $\Re$.

We cut off here the further description on the Dirichlet problem and shall continue it in Chap. IV.

\section{The types of Riemann surfaces}

Open abstract Riemann surfaces are classified into two types. Usually it is decided as follows :

Let $\Re$ be an open abstract Riemann surface, and $\left\{\Re_{n}\right\}$ its exhaustion with boundaries $\left\{C_{n}\right\}$. The harmonic measure of $C_{n}$ on the domains bounded by $C_{1}$ and $C_{n}$ is monotone decreasing as $n \rightarrow \infty$ and its limiting function is harmonic outside $\Re_{1}$. According as this is zero or positive, $\Re$ is said to have a null or positive boundary, or $\Re$ is called of parabolic or hyperbolic type respectively.

We will define the type of $\Re$ by Perron-Brelot's method using no exhaustion. Let $\Re_{0}$ be a domain of $\Re$, whose relative boundary $C$ consists of a finite number of simple closed analytic curves and whose complement is compact in $\Re$. We define the lower class $\mathfrak{u}$ by all the continuous subharmonic functions $\{u(P)\}$ on $\Re_{0}$ such that, $\Gamma_{\Re}$ denoting the ideal boundary of $\Re, \varlimsup_{\Gamma_{\Re}} u(P) \leqq 1^{32}$ and $\varlimsup_{c} u(P)$ $\leqq 0$, and $\underline{\omega}(P)$ is defined as the upper cover of $\mathfrak{u}$. Similarly the upper class is and its lower cover $\bar{\omega}(P)$ are defined for superharmonic functions. It follows by Lemma 1.1 that $\underline{\omega}(P)$ and $\bar{\omega}(P)$ are harmonic on $\Re_{0}$. Further wa can show

39) The left side denotes the supremum of $\varlimsup \lim u\left(P_{n}\right)$ for $P_{n} \rightarrow \Gamma_{\text {s. }}$. 
that $\underline{\omega}(P) \equiv \bar{\omega}(P)$ as follows: Let $C_{1}$ be a finite number of simple closed analytic curves on $\Re_{0}$ surrounding $C$. If we take an arbitrary $v(P) \in \mathfrak{B}$ and replace it in the annular domains bounded by $C$ and $C_{1}$ by the solution of the Dirichlet problem with the boundary value 0 on $C$ and $v(P)$ on $C_{1}$, the replacing function $v_{1}(P)$ is not greater than $v(P)$ and still belongs to $\mathfrak{B}$. Hence $0 \leqq \operatorname{\varlimsup im}_{C} \bar{\omega}(P)$ $\leqq \lim _{C} v_{1}(P)=0$. Consequently $\bar{\omega}(P) \in \mathfrak{U}$, whence $\bar{\omega}(P) \leqq \underline{\omega}(P)$. Since the inverse inequality is valid, there follows the equality.

It is a simple matter to show that this function coincides with the usual one. We shall call it the harmonic measure of $\Gamma_{\Re}$ on $\Re_{0}$ at $P$ and denote it by $\omega(P)$ or $\omega\left(P, \Gamma_{\mathfrak{R}}, \mathfrak{R}_{0}\right)$.

The property that $\omega(P) \equiv 0$ or $>0$ does not depend upon $\Re_{0}$ selected there and we decide the type of $\Re$ as usual. ${ }^{33)}$ The following is a criterion for it:

LEMMA 1.2, In order that $\Re$ has a positive boundary, it is necessary and sufficient that there exists a non-constant upper bounded continuous subharmonic. function on $\mathfrak{R}^{3.4)}$

Proof. If $\mathfrak{R}$ has a positive boundary, the function obtained by continuing the harmonic measure $\omega\left(P, \Gamma_{\Re}, \Re_{0}\right)$ by 0 outside $\Re_{0}$ is the required function, where $\Re_{0}$ is the domain taken to define the type of $\Re$.

Conversely suppose that such a function $u(P)$ exists. Without loss of generality it may be assumed that $u(P) \supseteqq 0$ and $\sup _{\Re} u(P)=1$. Since $u(P)$ is continuous subharmonic on $\Re, \sup _{\mathscr{R}_{0}} u(P)=1$ and $0 \leqq \max _{C} u(P)<1$, where $C$ is the boundary of $\Re_{0}$ in $\Re$. Thus $u(P)-\max _{C} u(P)$ belongs to the lower class $\mathfrak{U}$ and is positive at some point on $\Re_{0}$. Hence it follows that $\mathfrak{R}$ has a positive boundary.

Further we have

Lemma 1.3. Let $\Re$ have a null boundary and $\mathfrak{X}^{\prime}$ be a domain on it. In order that $\mathfrak{R}^{\prime}$ has a null boundary, it is necessary and sufficient that the transfinite kernel of $\mathfrak{\Re}-\mathfrak{X}^{\prime}$ on $\mathfrak{A}$ is empty. ${ }^{35)}$

Proof. Necessity: Suppose that the kernel $K$ is not empty, and take a small neighborhood $N^{36)}$ on $\Re$ of a boundary point of $K$ such that at least one point of $\mathfrak{R}^{\prime}$ lies outside $N$. Then we have a positive function in $N$, which is zero on the boundary of $N$, harmonic in $\mathfrak{R}^{\prime} \cap N$ and not greater than 1 . If we continue

33) When $\Re$ is a closed surface, it will also be said to have a null boundary.

34) It is reported in M. Parreau [3] that also M. Brelot has found the sufficiency.

35) This means that in every parameter circle the image of $\Re-\Re^{\prime}$ is of capacity zero.

36) We suppose in this paper that a neightorhood is bounded by a simple closed analytic curve. 
this function by 0 outside $N$, the function satisfies the condition in Lemma 1.2 and hence $\mathfrak{R}$ has a positive boundary.

Sufficiency: Suppose that the kernel is empty. Let $C$ be a simple closed analytic curve in $\Re^{\prime}$ which encloses a compact domain in $\mathfrak{R}^{\prime}$, and denote the non-compact part of $\mathfrak{N}^{\prime}$ by $\mathfrak{R}_{0}$. Since the image of $\mathfrak{R}-\mathfrak{R}^{\prime}$ in each parameter circle, corresponding to a neighborhood on $\Re$, is of capacity zero, it is removable for the function $\omega(P)=\omega\left(P, \Gamma_{\mathfrak{R}^{\prime}}, \mathfrak{R}_{0}\right)$, where $\Gamma_{\mathfrak{R}^{\prime}}$ is the ideal boundary of $\mathfrak{R}^{\prime}$. Hence $\omega(P)$ is defined so as to be bounded harmonic on $\mathfrak{R}$ outside $C$. Since $\omega(P)=0$ on $C$ and $\Re$ has a null boundary, $\omega(P) \equiv 0$. Thus $\Re^{\prime}$ has a null boundary.

Remark. When $\mathfrak{R}$ has a positive boundary, any domain on it has a positive boundary too on account of Lemma 1.2.

An open surface with null boundary has some properties as if it had no boundary. We shall state two of them for later uses.

LemMa 1.4. (Maximum principle). ${ }^{37)}$ Let $\mathfrak{R}$ have a null boundary, ${ }^{33)} \mathfrak{R}^{\prime}$ be a true subdomain with relative boundary $C$ on $\Re$. For a non-constant upper bounded continuous subharmonic function $u(P)$ on $\mathfrak{R}^{\prime}$ there holds

$$
u(P)<\varlimsup_{c} u(P) .
$$

Proof. Since $u(P)$ does not attain its maximum in $\Re^{\prime}$, it is sufficient to show $u(P) \leqq \varlimsup_{c} u(P)$. To the contrary suppose that there existed a point $P_{0}$ on $\Re^{\prime}$ such that $u\left(P_{0}\right)>\varlimsup_{c} u(P)$. Take a number $M$ between the numbers of both sides. The function $u_{1}(P)$, which is equal to $\max (u(P), M)$ on $\mathfrak{R}^{\prime}$ and to $M$ outside $\Re^{\prime}$, would be non-constant upper bounded continuous subharmonic on $\mathfrak{R}$. By Lemma $1.2 \Re$ would have a positive boundary.

LEMMA 1.5. Let $\mathfrak{R}$ have a null boundary, $\mathfrak{R}^{\prime}$ be a subdomain with relative boundary $C$ consisting of a finite number of simple closed analytic curves, and $u(P)$ be a bounded harmonic function on $\mathfrak{R}^{\prime}+C$. Then

$$
\int_{c} \frac{\partial u}{\partial \nu} d s=0 . \cdot^{39}
$$

Proof. This lemma is obvious when $\mathfrak{R}^{\prime}$ is compact in $\mathfrak{R}$. Hence suppose that $\mathfrak{R}^{\prime}$ is not compact in $\mathfrak{R}$ and let $\left\{\Re_{n}\right\}$ be an exhaustion of $\mathfrak{R}$. If $n$ is sufficiently large, $\mathfrak{R}^{\prime} \cap \Re_{n}$ is a domain bounded by $C$ and a part $\Gamma_{n}$ of the boundary

\footnotetext{
37) In R. Nevanlinna [5] this theorem was proved when a one-valued regular quadratically integrable differential $d w=d u+i d v$ is defined on $\Re$ and $u$ is its one-valued integral on $\Re$.

33) Cf. ${ }^{33)}$.

39) $\partial / \partial \nu$ represents the normal derivative inward to $\Re^{\prime}$.
} 
of $\Re_{n}$. By applying Green's formula for $u(P)$ and the harmonic measure $\omega_{n}(P)$ $=\omega\left(P, \Gamma_{n}, \mathfrak{R}^{\prime} \cap \Re_{n}\right)$, we have

$$
\int_{C+\Gamma_{n}} \frac{\partial \omega_{n}}{\partial \nu} d s=0
$$

and

$$
\int_{c+\Gamma_{n}}\left\{u \frac{\partial\left(1-\omega_{n}\right)}{\partial \nu}-\left(1-\omega_{n}\right) \frac{\partial u}{\partial \nu}\right\} d s=0
$$

whence follows

$$
\int_{C} \frac{\partial u}{\partial \nu} d s=-\int_{C+\Gamma_{n}} u \frac{\partial \omega_{n}}{\partial \nu} d s
$$

Since $u(P)$ is bounded: $|u(P)|<M<+\infty$, follows from (1.2) and (1.3)

$$
\int_{C} \frac{\partial u}{\partial \nu} d s \leqq M \int_{C+r_{n}} \frac{\partial \omega_{n}}{\partial \nu} d s=2 M \int_{C} \frac{\partial \omega_{n}}{\partial \nu} d s
$$

Because $\Re$ has a null boundary, $\omega_{n}(P) \downarrow 0$ and hence $\frac{\partial \omega_{n}}{\partial \nu} \downarrow 0$ as $n \rightarrow \infty$. Therefore from (1.4)

$$
\int_{c} \frac{\partial u}{\partial \nu} d s=0
$$

Remark. We took an exhaustion of $\mathfrak{A}$ only in proving Lemma 1.5, which will be used at the end of the proof of an existence theorem on $\Re$ with null boundary.

We shall give an outline of the proof, based upon the existence of a harmonic measure, for the fact that any Riemann surface $\mathfrak{i}$ is separable.

Let $\mathscr{D}$ and $\mathscr{D}^{\prime}$ be two disjoint domains which correspond to circular domains in a parameter circle, and denote the domain $\mathfrak{R}-\mathscr{D}-\mathfrak{D}^{\prime}$ by $\mathfrak{A}_{0}$. In order to prove the separability of $\Re$ it is sufficient to show it for $\Re_{0}$. The harmonic measure $\omega(P)$ of the boundary of $\mathscr{D}$ on $\Re_{0}$ is positive. If $\widetilde{\omega}(P)$ is its conjugate function, the function $w(P)=\omega(P)+i \widetilde{\omega}(P)$ is many-valued in general and its existence domain is a regular covering surface $\bar{\Re}$ over $\Re_{0}{ }^{40)}$ The inverse function $P=f(w)$ is many-valued too and its existence domain is a covering surface $\Re_{w}$ over the $w$-plane, to which $\overline{\mathfrak{R}}$ is conformally equivalent. It follows by the usual method that $\Re_{w}$ and hence $\bar{\Re}$ is separable. Then by the projection we can see that $\Re_{1}$ is separable too. It is a simple matter to obtain an exhaustion from an open base when $\mathfrak{R}$ is an open surface.

40) It is to be remarked that since the separability of $\mathfrak{R}$ is unknown, the basic surface $\mathscr{S}^{\prime \prime s}$ of $\overrightarrow{\mathfrak{R}}$ is not yet a surface in the usual meaning. 


\section{Chapter II. Existence Problems}

\section{Existence theorem on $\mathfrak{R}$ with positive boundary}

Our purpose in this chapter is to obtain a one-valued harmonic function, which has the singularity given in a compact part of $\mathfrak{R}$ and is bounded near the ideal boundary, if $\mathfrak{R}$ is open.41) We shall treat this problem distinguishing two cases; either the boundary of $\mathfrak{R}$ is null or positive. The proof will be based upon Perron-Brelot's method, in Theorem 2.1, and an idea due to M. H. Heins, ${ }^{42)}$ in Theorem 2.2. Hereafter in this chapter let $\mathfrak{R}_{0}$ be a domain, whose relative boundary $C$ consists of a finite number of simple closed analytic curves and whose complement is compact in $\mathfrak{R}$, and suppose that $\left(\mathfrak{R}_{0}^{a}\right)^{i}=\mathfrak{R}_{0}{ }^{43)}$ Further, put $\Re-\Re_{0}-C=\mathfrak{D}$ and let $U_{0}(P)$ be a one-valued function harmonic on $C$, that is, in an open set $\mathfrak{D}_{0}$ containing $C$.

When $\mathfrak{R}$ has a positive boundary, the problem can be solved always by

THEOREM 2.1.4) Let $\mathfrak{N}$ have a positive boundary $\Gamma_{\Re}$. Denote by $\mathfrak{U}$ the class, which will be called the lower class, of all the functions $\{u(P)\}$ such that each $u(P)$ is continuous and subharmonic on $\mathfrak{R}_{0} \cup \mathfrak{D}_{0}, u(P)-U_{0}(P)$ can be continued into $D$ preserving the subharmonic character and $\varlimsup_{\Gamma_{\Re}} u(P) \leqq 0$. Similarly the upper class $\mathfrak{B}$ is defined and both classes are not empty. The upper cover $U(P)$ of $\mathfrak{H}$ is equal to the lower cover $V(P)$ of $\mathfrak{B}$, is harmonic in $\Re_{0} \cup \mathfrak{D}_{0}$ and bounded on $\mathfrak{R}_{0}$, and the harmonic continuation of $U(P)-U_{0}(P)$ into $\mathfrak{D}$ is possible. If $\mathfrak{R}$ is contained in another Riemann surface $\mathfrak{R}$, then $\lim U(P)=0$ on the relative boundary of $\mathfrak{R}$ except at irregular points..$^{45}$

Proof. First we remark that, as is shown easily, when a function harmonic on $\mathfrak{D}+C$ is added to $U_{0}(P), \mathfrak{l}$ and $\mathfrak{B}$ do not change. Since $U_{0}(P)-H_{U_{0}}^{\mathfrak{D}}(P)$ is zero on $C$, it and hence $H_{C^{P}}^{\mathscr{P}}(P)$ is harmonic on $C$ by the principle of reflexion. Therefore the classes for $U_{0}(P)-H_{U_{0}}^{\mathfrak{D}}(P)$ are equal to $\mathfrak{U}$ and $\mathfrak{B}$. Hence we shall suppose that $U_{0}(P)$ itself is zero on $C$.

Next we shall show that $\mathfrak{H}$ and $\mathfrak{B}$ are not empty. ${ }^{46)}$ It is sufficient to show

41) R. Nevanlinna [8] discussed a wider problem, which includes our existence problem, from the viewpoint of integral equations.

42) M. H. Heins [1].

43) Suffixes $a$ and $i$ indicate to take the closure and the open kernel respectively.

44) We get the function $U(P)$ in this theorem also by alternierendes Verfahren.

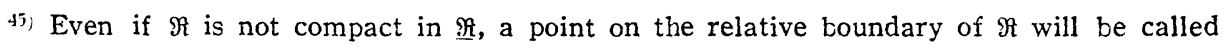
regular or irregular if its image is so in a local parameter circle corresponding to a neighborhood on $\mathfrak{\Re}$.

46) The idea is indebted to M. Brelot [4]. 
it for $\mathfrak{H}$, because if $u(P)$ belongs to the lower class for $-U_{0}(P)$ then $-u(P)$ belongs to the upper class for $U_{0}(P)$. Since $\mathfrak{R}$ has a positive boundary, $\omega(P$, $\left.\Gamma_{\Re}, \Re_{0}\right)=\omega(P)>0$ on $\Re_{0}$. Since $\omega(P)=0$ on $C$ it is harmonic also on $C$ by the principle of reflexion. Let $\Delta:|t|<1(t=x+i y)$ be a local parameter circle, which corresponds to a neighborhood on $\Re$ and in which the image of $\Re_{0}$ is equal to the upper semicircular domain. Then the composed function $\omega(P(t))$ is harmonic in $\Delta$ and $\frac{\partial \omega}{\partial y}>0$ on $-1<x<1$. Hence if $y_{0}$ is taken sufficiently small, $\frac{\partial \omega}{\partial y}>\alpha>0$ and $\left|\frac{\partial U_{0}}{\partial y}\right|<K<+\infty$ on the rectangle $A:-1+\varepsilon \leqq x \leqq 1-\varepsilon,-y_{0} \leqq y \leqq 0$. Taking a finite number $K_{1}>\frac{K}{\alpha}$, we have for $y\left(0<y \leqq y_{0}\right)$

$$
-K_{1}+U_{0}+K_{1}(1-\omega)=\int_{-y}^{0}\left(-\frac{\partial U_{0}}{\partial \eta}+K_{1} \frac{\partial \omega}{\partial \eta}\right) d \eta>\left(-K+\alpha K_{1}\right) y>0 .
$$

Hence $-K_{1}+U_{0} \geqq-K_{1}(1-\omega)$ on $A$. Since $C$ is covered by a finite number of images of such rectangles, the function defined by $-K_{0}+U_{0}$ on $\mathfrak{D}_{0} \cap \mathfrak{D}$ and by $K_{0}(\omega-1)$ on $\mathfrak{R}_{0}$ is subharmonic also on $C$ and belongs to $\mathfrak{u}$, if $K_{0}$ is taken sufficiently large. Clearly this function is bounded on $\Re_{0}$.

Since any $u(P) \in \mathfrak{U}$ is not greater than any $v(P) \in \mathfrak{B}$, there holds $U(P)$ $\leqq V(P)$ and both are bounded on $\mathfrak{R}_{0}$. Further they are harmonic on $\Re_{0} \cup \mathfrak{D}_{0}$ on account of Lemma 1.1. Similarly we can show that $U(P)-U_{0}(P)$ and $V(P)$ $-U_{0}(P)$ are harmonically prolongable into $\mathfrak{D}$.

Now we shall show that $U(P) \equiv V(P)$. Since $D$ is bounded by a finite number of simple closed analytic curves, it consists of a finite number of compact domains. Excluding a smaller compact domain from each component, define harmonic measures there respectively. Thus we have a positive function $H(P)$ which is harmonic near $C$ in $\mathscr{D}$ and zero on $C$. For a number $k>0$ denote by $U_{0}^{+}(P)$ and $U_{0}^{-}(P)$ the functions which are equal to $U_{0}(P)+k H(P)$ and $k H(P)$ respectively. Then both are harmonic also on $C$ and $U_{0}(P)=U_{0}^{+}(P)-U_{0}^{-}(P)$, and, for large $k, U_{0}^{+}(P)>0$ near $C$ in $\mathscr{D}$. Taking $U_{0}^{+}(P)$ and $U_{0}^{-}(P)$ instead of $U_{0}(P)$ used hitherto, we obtain the lower and upper classes $\mathfrak{H}^{+}, \mathfrak{H}^{-}, \mathfrak{B}^{+}, \mathfrak{V}^{-}$and their covers $U^{+}(P), U^{-}(P), V^{+}(P), V^{-}(P)$ respectively. If we define a func. tion by 0 on $\Re_{0}$ and by $U_{0}^{+}(P)$ on $\mathfrak{D}_{0} \cap \mathfrak{D}$ it belongs to $\mathfrak{H}^{+}$. Therefore $U^{+}(P)$ $\triangleq 0$ on $\mathfrak{R}_{0}$ and hence $E \mathfrak{B}^{+}$. Thus $U^{+}(P) \triangleq V^{+}(P)$. Since the converse is obvious, there holds the equality. Similarly we have $U^{-}(P)=V^{-}(P)$. Let $u^{+}, u^{-}, v^{+}, v^{-}$ be any functions belonging to $\mathfrak{U}^{+}, \mathfrak{H}^{-}, \mathfrak{B}^{+}, \mathfrak{F}^{-}$respectively. Then $u^{+}-v^{-} \in \mathfrak{l}$ and $v^{+}-u^{-} \in \mathfrak{B}$, and hence $u^{+}-v^{-} \leqq U \leqq V \leqq v^{+}-u^{-}$. Since $u^{+}$and $u^{-}$can be chosen arbitrarily near $v^{+}$and $v^{-}$respectively, we have $U(P) \equiv V(P)$.

When $\mathfrak{R} \cup \mathfrak{R}$ and $N$ is a neighborhood on $\Re$ of a point of the relative boundary of $\mathfrak{R}, U(P)$ coincides with the general solution of the Dirichlet problem on $N \cap \mathfrak{R}$ 
with the boundary value 0 on $\Gamma_{\Re}$ and $U(P)$ in $\Re$. Therefore $\lim U(P)=0$ on the relative boundary of $\Re$ except at irregular points. Thus the proof is completed.

In the sequel we shall call $U(P)$ the solution for $U_{0}(P)$.

2. Existence theorem on $\Re$ with null boundary

In this case we can solve the problem in the following form:

TheORem 2.2.4i) Let $\Re$ have a null boundary and choose any point $P_{0}$ on $\Re_{0}$. Then there exists a one-valued harmonic function $U(P)$ on $\Re_{0} \cup \mathfrak{D}_{0}-\left\{P_{0}\right\}$ such that it is bounded on $\mathfrak{R}_{0}$ except near $P_{0}$, the harmonic continuation into $\mathfrak{D}$ of $U(P)-U_{0}(P)$ is possible and the development of $U(P)$ in a local parameter circle $\Delta:|t|<1$, whose centre corresponds to $P_{0}$, has the form:

$$
a_{0} \log \frac{1}{|t|}+a \text { harmonic function, }{ }^{43)}
$$

where $a_{0}=\frac{1}{2 \pi} \int_{c} \frac{\partial U_{0}}{\partial \nu} d s^{49}$ The function $U(P)$ is determined uniquely up to an additional constant.

Proof. Let $C_{n}$ be the image on $\Re$ of the circle $|t|=\frac{1}{n}$ in $\Delta$, and $\Re_{n}$ the domain outside $C_{n}$ on $\Re$. Since $\Re_{n}$ has a positive boundary, the solution $U_{n}(P)$ for $U_{0}(P)$ exists on $\Re_{n}$ for each $n$. by theorem 2.1 .

In the first place suppose that there exists an infinite subsequence $\left\{U_{n(i)}(P)\right\}$ such that $\max _{c} U_{n(i)}(P) \geqq 0$. If we denote by $V_{i}(P)$ the function $U_{n i i}(P)$ $-\max _{c} U_{n(i)}(P)$, then $V_{i}(P) \leqq 0$ on $\Re_{n(\imath)} \cap \Re_{0}$ by Lemma 1.4 and $\max _{c} V_{i}(P)=0$. Further it follows that $\max _{C_{1}} V_{i}(P) \geqslant-\max _{c} U_{1}(P)$ for every $i$ on account of Lemma 1.4. Therefore we can extract from $\left\{V_{i}(P)\right\}$ a subsequence which converges uniformly in the wider sense on $\Re_{0}-\left\{P_{0}\right\}$ to a one-valued harmonic function $U(P)$ which is non-positive on $\Re_{0}$. Hence $U(P)$ is developed in $\Delta$ in the form:

$$
a_{0} \log \frac{1}{|t|}+\text { a harmonic function } \quad\left(a_{0} \leqq 0\right) .
$$

In addition, $U(P)$ is bounded on $\Re_{1} \cap \Re_{0}$, because $V_{i}(P) \supseteqq \min _{C+C_{1}} V_{i}(P)$ by Lemma

4i) In case $a_{0}=0$ this theorem may be proved by alternierendes Verfahren as stated in $R$. Nevanlinna [5], L. Sario [1], or by the method used in K. Kodaira [1] in a special case. The general case of our form can be deduced from the case: $a_{0}=0$ without difficulty.

43) We shall call this a logarithmic singularity of $U(P)$ when $a_{0} \neq 0$. $a_{0}$ will be called the residue of $U(P)$ at $P_{0}$.

49) The normal $\nu$ is drawn inward to $\Re_{0}$. 
1.4 and the convergence is uniform on $C+C_{1}$. Further it follows without difficulty that the harmonic continuation into $\mathscr{D}$ of $U(P)-U_{0}(P)$ is possible.

When there is no infinite subsequence stated above, consider the functions $\left\{-U_{n}(P)\right\}$ and treat them in the same manner. By changing again the sign of the limiting function we have a function $U(P)$ with the development:

$$
a_{0} \log \frac{1}{|t|}+\text { a harmonic function } \quad\left(a_{0} \geqq 0\right) \text {. }
$$

According to Lemma $1.5 \int_{C+c_{1}} \frac{\partial U}{\partial \nu} d s=0$. Hence $a_{0}=-\frac{1}{2 \pi} \int_{C_{1}} \frac{\partial U}{\partial \nu} d s=\frac{1}{2 \pi} \int_{C} \frac{\partial U}{\partial \nu} d s$ $=\frac{1}{2 \pi} \int_{c} \frac{\partial U_{0}}{\partial \nu} d s$.

The uniqueness of $U(P)$ is the direct consequence of Lemma 1.4.

\section{Variation of preassigned singularities}

We shall answer to the problem how the solution $U(P)$ varies as the preassigned function $U_{0}(P)$ does, by

THEOREM 2.3. When $U_{0}^{(i)}(P)$ tends to $U_{0}(P)$ uniformly in an open set $\mathscr{D}_{0}$ $\supset C$ as $i \rightarrow \infty$, the solution $U_{i}(P)$ for $U_{0}^{(i)}(P)$ tends to the solution $U(P)$ for $U_{0}(P)$ uniformly on $\Re_{0}$ when $\Re$ has a positive boundary. When $\mathfrak{M}$ has a null boundary, designate $a$ point $P_{0}$ to the common possible logarithmic singular point and normalize all the solutions by $U_{i}\left(P_{1}\right)=U\left(P_{1}\right)$ at a point $P_{1}\left(\neq P_{0}\right) \in \Re_{0}$. Then the convergence is uniform on any closed set in $\Re_{0}-\left\{P_{0}\right\}$.

Proof. First consider the case when $\mathfrak{R}$ has a positive boundary. Without loss of generality we may suppose $U_{0}^{(i)}(P)=U_{0}(P)=0$ on $C$. For any $u(P) \in \mathfrak{H}$ define a function by $u(P)-K_{i}(1-\omega(P))$ on $\Re_{0}$ and $u(P)-U_{0}(P)+U_{0}^{(i)}(P)-K_{i}$ on $\mathscr{D} \cap \mathscr{D}_{0}$, where $K_{i}>0$ is a constant. If $K_{i}$ is chosen suitably, this function is subharmonic also on $C$ and hence belongs to the lower class for $U_{0}^{i)}(P)$. Especially on $\Re_{0}+C$ there holds $u(P)-K_{i}(1-\omega(P)) \leqq U_{i}(P)$. Since $u(P)$ is taken arbitrarily near $U(P)$, follows $U(P)-K_{i} \leqq U_{i}(P)$ on $\mathfrak{R}_{0}+C$. Similarly we can show $U_{i}(P)-K_{i}^{\prime} \leqq U(P)$ on $\Re_{0}+C$, where $K_{i}^{\prime}$ is a positive constant. Hence $\left|U(P)-U_{i}(P)\right| \leqq K_{i}+K_{i}^{\prime}$ on $\mathfrak{R}_{0}+C$. Since $U_{0}^{(i)}(P)$ tends to $U_{0}(P)$ uniformly in $\mathfrak{D}_{0}$, we may take $K_{i}$ and $K_{i}{ }^{\prime}$ arbitrarily small as $i \rightarrow \infty$. Consequently $U_{i}(P)$ tends to $U(P)$ uniformly on $\mathfrak{N}_{0}+C$.

Next we shall treat the case when $\Re$ has a null boundary. We shall denote the solution for $U_{0}^{(i)}(P)$ on the domain $\Re_{1}$ outside $C_{1}{ }^{\text {in }}$ by $W_{i}(P)$. Since $\Re_{1}$ has a positive boundary, $W_{i}(P)$ converges uniformly on $C$. Hence $\left|W_{i}(P)\right|<\alpha$ $<+\infty$ on $C$ for all $i$. In case there exists an infinite subsequence $\left\{a_{i, j}\right\}$ with

50) We use notations in the proof of Theorem 2.2. 
$a_{i(j)} \leqq 0$, we define functions $\left\{X_{j}(P)\right\}$ by $X_{j}(P)=U_{i(j)}(P)-\max _{c} U_{i(j)}(P)$. Then $X_{j}(P) \leqq 0$ on $\Re_{0}$ by Lemma 1.4 and $\max _{c} X_{j}(P)=0$. Further there follows $\max _{(1} X_{j}(P) \supseteqq-\max _{c} W_{i(j)}(P)>-\alpha$. Therefore a subsequence $\left\{X_{j(k)}(P)\right\}$ converges to a function $X(P)$ uniformly on any closed set in $\Re_{0}-\left\{P_{0}\right\}$. We can see easily that $X(P)$ is a solution for $U_{0}(P)$. By the uniqueness of the solution there holds $X(P)-X\left(P_{1}\right)=U(P)-U\left(P_{1}\right)$. Since $\max _{C} U_{i(j(k))}(P)=U_{i(j(k))}\left(P_{1}\right)-X_{j(k)}\left(P_{1}\right)$ tends to $U\left(P_{1}\right)-X\left(P_{1}\right), \quad U_{i(j(k))}(P)=X_{j(k)}(P)+\max _{c} U_{i(j(k))}(P)$ tends to $X(P)$ $+U\left(P_{1}\right)-X\left(P_{1}\right)=U(P)$ uniformly on any closed set in $\mathfrak{R}_{0}-\left\{P_{0}\right\}$. In case almost all $a_{i}>0$ consider the functions $\left\{-U_{i}(P)\right\}$ and treat them in the same manner. By changing the sign again we know that a subsequence of $\left\{U_{i}(P)\right\}$ converges to $U(P)$ uniformly on any closed set in $\mathfrak{R}_{0}-\left\{P_{0}\right\}$. Because from any subsequence of $\left\{U_{i}(P)\right\}$ we can extract a convergent subsequence, $U_{i}(P)$ itself converges to $U(P)$ uniformly on any closed set in $\Re_{0}-\left\{P_{0}\right\}$.

\section{The simplest harmonic functions on $\mathfrak{\Re}$.}

Given a Riemann surface $\mathfrak{R}$ we will decide the simplest function on it, which is one-valued harmonic outside isolated singularities and bounded near the ideal boundary.

Now suppose that $\mathfrak{R}$ has a null boundary. By Lemma 1.2 there does not exist not only bounded but also positive non-constant harmonic function on $\mathfrak{R}$. Moreover no Green function exists on $\mathfrak{R}$, because if the Green function $G(P)$ existed on $\Re,-\min (G(P), M)$ for a constant $M>0$ would be a non-constant negative continuous subharmonic function on $\Re$ and hence by Lemma $1.2 \Re$ would have a positive boundary. The simplest function is the one with two logarithmic singularities or a non-logarithmic singularity of the local form: $\frac{\cos \theta}{r}$ or $\frac{\sin \theta}{r}$. The existence of these functions is assured by Theorem 2.2.

Next suppose that $\Re$ has a positive boundary. It is reported that L. Ahlfors found a surface with positive boundary on which no non-constant bounded harmonic function exists. ${ }^{51}$ But it is unknown whether there exists or not a surface with positive boundary on which no non-constant positive harmonic function exists.52) The existence of the function having any assigned singularities is assured by Theorem 2.1; especially, the Green function exists always there. ${ }^{53)}$

51) Cf. L. Sario [2], K. I. Virtanen [1], R. Nevanlinna [8].

52) M. Parreau [3] presented this question.

53) In P. J. Myrberg [1] this was proved in a special case. R. Nevanlinna [3], [4] stated that the Green function exists if and only if the boundary of $\Re$ is positive. A proof to it is given above and proofs are found also in L. Sario [2], M. Parreau [1], K. I. Virtanen [1]. 


\section{Chapter III. Boundary Correspondences in Conformal Mappings}

\section{One-to-one correspondence between boundaries}

In the former haif $(\$ \S 1-2)$ of this chapter we shall investigate the image of the accessible boundary points (which will be abbreviated by A. B.P.s), when the universal covering surface $\Re^{\infty}$ of an open covering surface $\Re$ over a Riemann surface $\Re$ is mapped conformally onto a unit circular domain $U:|z|<1$, under the following conditions :

(A) the projection $\underline{\Re}^{\prime}$ of $\mathfrak{R}$ is compact in $\mathfrak{R} ;{ }^{54)}$

(C) when $\mathfrak{R}$ is a closed surface of genus zero or one, $\mathfrak{R}-\mathfrak{R}^{\prime}$ contains at least three or one point respectively.

When $\Re$ is a covering surface over a Riemann sphere $\sigma_{0}$, O. Teichmüller introduced a metric on $\mathfrak{R}$ by inf $\delta\left(\widehat{P}_{1} \widehat{P}_{2}\right)$, where $\delta\left(\widetilde{P_{1} P_{0}}\right)$ is the diameter of the projection into $\sigma_{0}$ of $\widehat{P}_{1} P_{2}$ which connects $P_{1}$ with $P_{2}$ on $\mathfrak{R}$, and he defined A.B.P.s of $\Re$ by the completion of $\Re$ with respect to this metric. ${ }^{53)}$ Now we consider any inner covering surface $\Re$ over $\mathfrak{R}$. Since there exists always a non-constant one-valued meromorphic function on $\Re^{56)} \mathfrak{R}$ is conformally equivalent to a covering surface over $\sigma_{0}$. Regarding $\Re$ itself as a covering surface over $\sigma_{0}$, a metric and A.B.P.s are defined as above. We shall call this metric a Teichmüller's metric, these A.B.P.s A.B.P.s in the sense of Teichmüller, and denote all the A.B.P.s by $\mathfrak{I}_{\mathfrak{R}}$. We can give their definition in an equivalent way by deciding the equivalency of two curves which determine A.B.P.s.

On the other hand $R$. Nevanlinna limited further the range of the equivalency of two determining curves and defined A.B.P.s of different kind.57) Using this definition he investigated the image on $\Gamma:|z|=1$ of the A.B.P.s, when $\Re$ is a compact domain in $\mathfrak{R}$. Similarly we can prove the following theorems, ${ }^{51}$ in which A.B.P.s mean those in the sense of Nevanlinna, under the conditions (A) and (C):

THEORFM 3.1. The images of the curves, which determine one and the same A.B.P. of $\Re$, terminate at points on $\Gamma$ which are equivalent with respect to a Fuchsian group. However, if two curves determine different A.B.P.s, the images terminate at no same point on $\Gamma$.

\footnotetext{
54) See introduction. We shall call a covering surface satisfying the condition (A) an inner covering surface.

55) O. Teichmüller [1].

;i) Cf. J. Tamura [1], for instance.

5i) R. Nevanlinna [1], [3]. Cf. also E; Kaila [1].

is) Cf. R. Nevanlinna [3], E. Kaila [1].
} 
THEOREM 3.2. If $z$ tends to $a$ point on $\Gamma$, which corresponds to an A.B.P. of $\Re$, along any Stolz's path, the image of the path determines the same A.B.P. of $\Re$.

Theorem 3.1 shows that all the A.B.P.s and the classes of points corresponding to them correspond to each other in a one-to-one manner. Therein lies the reason why A.B.P.s are defined in Nevanlinna's sense here. But the image on $T$ of all the A.B.P.s is the same by either of two definitions. If we accept A.B.P.s in Teichmüller's sense, the mapping of $\mathfrak{I}_{\mathfrak{F}} \infty$ into $\Gamma$ is uniformly continuous with respect to the Teichmüller's metric.9?

\section{Measure of the image of the accessible boundary points}

$R$. Nevanlinna stated that, when $\mathfrak{R}$ is compact in $\mathfrak{R}$, the linear measure of the image on $T$ of all the A.B.P.s of $\Re$ is 0 or $2 \pi$ according as the transfinite kernel of $\underline{R}-\Re$ on $\underline{R}$ is empty or not, and that the proof is similar to the case when $\mathfrak{R}$ is a plane domain. ${ }^{601}$ We shall give generalizations of this result for $\mathfrak{R}$ satisfying the conditions (A) and (C).

First we will prove

LEMMA 3.1. For any continuum $E$ on a plane there exists a totally.disconnected closed subset of positive capacity.

Proof. If $E$ contains a segment, let the segment be $\overline{P Q}$. Otherwise take two points $P$ and $Q$ on $E$. We may suppose that $\overline{P Q}=\overline{01}$ on the $x$-axis. The set of all the irrational points on $\overline{01}$ is of positive capacity, and hence has a closed subset $E_{1}$ of positive capacity. If $\overline{01} \subset E, E_{1}$ is the required set. Otherwise draw orthogonal lines to the $x$-axis at every point of $E_{1}$ and let $E^{\prime}$ be the intersection of these lines with $E$. Then $E^{\prime}$ is closed and totally-disconnected, since both sets are closed, $E_{1}$ is totally-disconnected and $E$ contains no segment. Further the capacity of $E^{\prime}$ is positive, because the capacity of a set is greater than that of its projection on a line. Thus we have a required set.

Using this lemma we can show

THEOREM 3.3. If $\mathfrak{R}$ satisfies $(A)$ and if the transfinite kernel of $\mathfrak{R}-\mathfrak{R}^{\prime}$ is non-empty, the linear measure of the image on $\Gamma$ of all the A.B.P.s of $\Re$ is $2 \pi$.

Proof. On account of Lemma 3.1 there exists a closed totally-disconnected true subset $E$, having a non-empty transfinite kernel, of $\underline{\Re}-\underline{\Re}$. By Lemma 1.3 $\mathscr{R}-E$ has a positive boundary and hence there exists the Green function $G(P)$ on $\mathfrak{R}-E$ with a pole at a point $Q_{0}$ of $\underline{\Re}-\underline{\mathbb{R}}^{\prime}-E$. Let $H(P)$ be the conjugate

59) Cf. O. Teichmüller [1].

6in) R. Nevanlinna [3]. Cf. also M. Tsuji [4]. 
function of $G(P)$ on $\mathscr{R}-E$ and put $\exp (-G(P)-i H(P))=W(P)$. Compose the mappings $U \rightarrow \Re \rightarrow \Re \rightarrow W$-plane and denote the corresponding function by $W=g(z)$, taking an arbitrary branch of $W(P)$. Then $g(z)$ is a bounded regular function in $U$ and hence there exist radial limits a.e. ${ }^{61)}$ on $I$ by Fatou's theorem.

We shall prove that if $g(z)$ tends to a limit $W_{0}$ on the $W$-plane along a radius terminating at a point on $\Gamma$, the image $C$ on $\mathfrak{R}$ of the radius terminates at a point on $\mathfrak{R}$, i.e., the image on $\mathfrak{R}$ determines an A.B.P.

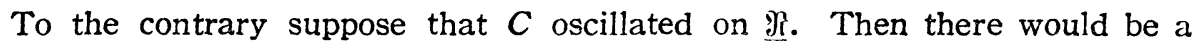
point $P_{0}$ of $\Re-E-\left\{Q_{0}\right\}$ and its neighborhood $N$ in $\Re-E-\left\{Q_{0}\right\}$ such that $C$ would accumulate on $P_{0}$ and intersect the boundary of $N$ infinitely often, since $E$ is totally-disconnected. Let a local parameter circle corresponding to $N$ be $\Delta:|t|<1$, where $t=0$ corresponds to $P_{0}$. The image of $C$ in it consists of an enumerably infinite number of $\operatorname{arcs}\left\{l_{n}\right\}$ passing nearer to $t=0$ as $n \rightarrow \infty$. If

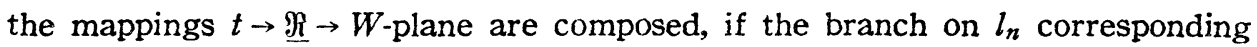
to the function $g(z)$ is continued analytically everywhere in $\Delta$ and if the branch obtained is denoted by $W_{n}(t)$, then $W_{n}(t)$ would tend to $W_{0}$ uniformly on $l_{n}$ as $n \rightarrow \infty$. Since $\left|W_{n}(t)\right|<1,\left\{W_{n}(t)\right\}$ would form a normal family in $\Delta$ and hence we could extract a subsequence $\left\{W_{n(2)}(t)\right\}$ tending to a regular function in $\Delta$ as $i \rightarrow \infty$. Because $W_{n(i)}(t) \rightarrow W_{0}$ uniformly on $l_{n(i)}$, which would come near $t=0$ but would be a cross-cut of $\Delta$, the limiting function would be equal to the constant $W_{0}$. However, $\left|W_{n(i)}(t)\right|=\exp (-G(P))$ independently of $i$ and so this is not a constant. Thus we have a contradiction and it is proved that $C$ does

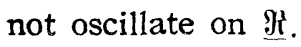

We will say that an inner covering surface $\mathfrak{R}$ over $\mathfrak{R}$ is of $F$-type when the function corresponding to the mappings $U \rightarrow \mathfrak{N}^{\infty} \rightarrow \mathfrak{R} \rightarrow \mathfrak{R}$ has limits along Stolz's paths a.e. on $\Gamma$. Theorem 3.3 gives a condition so that $\Re$ is of F-type.

M. Tsuji has given the following interesting extension of Lusin-Priwaloff's theorem. ${ }^{62}$ His proof will be simplified a little by using the terms of Dirichlet problem.

LemMa 3.2. Let $w=F(z)$ be a meromorphic function in $U, E$ a measurable set of positive linear measure on $\Gamma$ and $E^{\prime}$ a set of (inner) capacity zero on the w-plane. If the cluster set of $F(z)$, when $z$ tends to points of $E$ along Stolz's paths, is contained in $E^{\prime}$, then $F(z)$ is a constant belonging to $E^{\prime}$.

Proof. Since $E^{\prime}$ is of inner capacity zero, it does not contain any continuum and hence $F(z)$ has a limit: $F\left(e^{i \theta}\right) \in E^{\prime}$ along Stolz's paths at every point

61) This is an abbreviation of "almost everywhere."

62) N. Lusin and J. Priwaloff [1]. M. Tsuji [3]. 
$e^{i \theta}$ of $E$.

In a usual way we get a domain $D$, whose boundary is a rectifiable curve $C$ consisting of a closed subset $E_{1}$, with a positive linear measure, of $E$ and segments in $U$, such that the number of the poles on $D+C$ of $F(z)$ is finite and $E_{1}^{\prime}=\left\{F\left(e^{i \theta}\right) ; e^{i \theta} \in E_{1}\right\}$ is a bounded closed set in the $w$-plane. Further exclude the poles by cross-cuts from $D+C$ such that the remaining domain $D_{1}$ is bounded by a rectifiable Jordan curve $C_{1}$ and $E_{1}$ lies on $C_{1}$. Then the harmonic measure on $D_{1}$ of $E_{1}$ is positive on account of $F$. and M. Riesz's theorem. ${ }^{63}$

Now let $v(w)$ be the potential due to G. C. Evans ${ }^{(4)}$ in the $w$-plane. It is induced by a mass distributed on $E_{1}^{\prime}$ and takes $+\infty$ on $E_{1}^{\prime}$. We shall denote the composed function $v(F(z))$ by $V(z)$. When $V(z) \neq+\infty$, this is superharmonic in $D_{1}$ and tends to $+\infty$ when $z \rightarrow E_{1}$ from the inside of $D_{1}$. Further there exists a number $\alpha>-\infty$ such that $V(z) \geqslant \alpha$ in $D_{1}$. If a boundary function $\varphi$ of $D_{1}$ is given by $\lim V$ on $C_{1}, V(z)$ belongs to the upper class $\mathfrak{B}_{\varphi}^{D_{1}},{ }^{6 j}$ irrespective of whether $V(z) \equiv+\infty$ or not, and hence there holds $H_{\varphi}^{n_{1}}(z) \leqq V(z)$ in $D_{1}$. Since the harmonic measure of $E_{3}$ with respect to $D_{1}$ is positive and $\varphi=+\infty$ on $E_{1}, H_{\varphi}^{D_{1}}(z) \equiv+\infty$ and hence $V(z) \equiv+\infty$. This means that $w=F(z)$ is a constant belonging to $E^{\prime}$ and the theorem is proved.

From this lemma we get easily

Theorem 3.4. Suppose that $\mathfrak{R}$ satisfies $(A)$ and $(C)$ and let $(5$ be a subset of $\mathfrak{I}_{\Re}$. If the transfinite kernel of any closed subset of the projection on $\mathfrak{R}$ of $\mathbb{E}$ is empty, the linear inner measure of the image $E$ on $\Gamma$ of $E$ is zero.

Proof. There exists always a non-constant one-valued meromorphic function

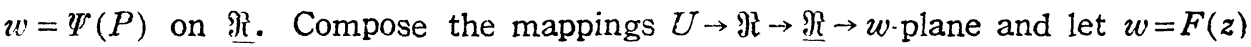
be the corresponding function in $U$. According to Theorem $3.2 F(z)$ has a limit along Stolz's paths at every point of $E$ and the set $\left\{\lim _{E} F(z)\right\}$ is of inner capacity zero on the $w$-plane. On account of Lemma 3.2 the measure of any measurable subset of $E$ is zero, whence the inner measure of $E$ is zero.

Remark. If $\mathfrak{F}=\mathfrak{I}_{\mathfrak{R}}$ then $E$ is measurable, because it is the continuous image of the complete metric space $\mathfrak{I}_{\mathfrak{R}}$, and hence an analytic set.

This and Theorem 3.3 can be regarded as generalizations of Nevanlinna's result.

\footnotetext{
63) F. and M. Riesz [1]. See Chap. V, $\S 5$ of our present paper.

64) G. C. Evans [1].

65) We suppose here that the upper class includes non-continuous superharmonic functions.
} 
3. Mapping of an abstract Riemann surface

Suppose that the universal covering surface of an abstract Riemann surface $\Re$ is mapped conformally onto $U:|z|<1$. Then $U$ is divided as usual into non-overlapping fundamental domains in the following manner: ${ }^{66}$ )

Let $\left\{a_{n}\right\}$ be a class of points corresponding to a point $P_{0} \in \Re$ and $\bar{U}_{n}$ be the set of points $\{z\}$ in $U$ such that

$$
\rho\left(a_{n}, z\right) \leqq \rho\left(a_{k}, z\right) \text { for all } a_{k} \quad(k \neq n),
$$

where $\rho\left(z_{1}, z_{2}\right)$ represents the non-euclidean distance $\left|\frac{z_{1}-z_{2}}{1-\bar{z}_{1} z_{2}}\right| \cdot \bar{U}_{n}$ is bounded by at most an enumerably infinite number of circular arcs perpendicular to $\Gamma$ : $|z|=1^{6 i}$ and possibly by a part of $T$, and is circular convex. The boundary of $\bar{U}_{n}$ in $U$ is divided into pairs of equivalent sides and by excluding one side of every pair a fundamental domain $U_{n}$ is obtained. This will be called a normal polygon with centre $a_{n}$ and denoted by N.P. All the $\left\{U_{n}\right\}$ cover $U$ without any gap.

We shall investigate in detail the boundary correspondence in the case when $\Re$ is a non-simply-connected open abstract Riemann surface, whose universal covering surface is of hyperbolic type, in the sequel. In this case, every N.P. is non-compact in $U$.

\section{Regular points}

The cluster set $S_{z_{0}}^{(U)}$ of $f(z)$, which is the mapping function of $U$ onto $\Re$, at a point $z_{0}$ on $\Gamma$ will be defined as the set of all the points $\{P\}$ of $\Re$ for each of which there exists a sequence $\left\{z_{k}\right\}$ tending to $z_{0}$ in $U$ such that $f\left(z_{k}\right) \rightarrow P$ as $k \rightarrow \infty$. A point $z_{0} \in T$ will be called regular or singular according as $S_{z_{0}}^{(U)}=\phi$ or $\neq \phi{ }^{63)}$ Since fixed points of non-trivial linear substitutions of the Fuchsian group $(\mathfrak{B}$, with respect to which $f(z)$ is automorphic, are singular, at least one singular point always appears, but regular points do not necessarily as in the case when $f(z)$ is a modular function.

The intersection of a circular domain with centre $z_{0}$ with $U$ will be called a vicinity of $z_{0}$ in $U$.

We shall investigate the regular points in this section. First we will prove

THEOREM 3.5. Let $z_{0}$ be a regular point on $T$. Then we can take a vicinity of $z_{0}$ in $U$ such that $f(z)$ is univalen there.

55) Cf. H. Weyl [1], pp. 154-156, L. Bieberbach [1], 1 Aufl., pp. 45-53.

67) We will call these arcs the sides. They are non-euclidean (N.E.) segments, or halflines, or lines.

63) $\phi$ denotes empty set. 
Proof. If there were a infinite number of sides in any vicinity of $z_{0}, z_{0}$ would be a singular point..$^{(9)}$ Hence if a vicinity $V$ of $z_{0}$ is taken sufficiently small, either there is no side of N.P. in $V$ or $V$ is divided into a finite number of wedge-shaped parts of N.P.s with $z_{0}$ as a common vertex. In the former case $f(z)$ is univalent in $V$. In the latter case suppose that $f(z)$ were not univalent in any vicinity of $z_{0}$. Then there would be two disjoint infinite sequences $\left\{z_{k}\right\}$ and $\left\{z_{k}^{\prime}\right\}$ tending to $z_{0}$ such that $f\left(z_{k}\right)=f\left(z_{k}^{\prime}\right)$. We could find infinite subsequences $\left\{z_{k(i)}\right\}$ and $\left\{z_{k(i)}^{\prime}\right\}$ in some two of the wedge-shaped parts of N.P.s: $N$ and $N^{\prime}$ respectively. The linear substitution which transforms $N$ to $N^{\prime}$ would leave $z_{0}$ fixed. This is a contradiction, because fixed points are singular. Thus $f(z)$ is univalent in a vicinity of $z_{0}$.

The following lemma will be used often:

LEMMA 3.3. Let $\gamma$ be an inscribed circle passing through $a_{0} \in U$ and touching $\Gamma$ at $z_{0}$. According as a point $a_{0}^{\prime} \neq a_{0}$ in $U$ lies inside, or upon, or outside $r$, the $N . E$. line equidistant from $a_{0}$ and $a_{0}^{\prime}$ intersects the N.E. half line $\widehat{a_{0} z_{0}}$, or passes through $z_{0}$, or is disjoint to $\widehat{a_{0} z_{0}}$ respectively.

Proof. We may suppose without loss of generality that $a_{0}=0$ and $z_{0}=1$. Put $a_{0}^{\prime}=r e^{i \theta}$ and let $z=\rho e^{i \varphi_{\rho}}(0<\rho<1)$ be the point equidistant from $a_{0}^{\prime}$ and 0 . Then it follows from $\rho=\left|\frac{a_{0}-z}{1-\bar{a}_{0} z}\right|$ that

$$
r^{2}\left(1-\rho^{4}\right)-2 r \rho\left(1-\rho^{2}\right) \cos (\theta-\varphi)=0 \text {. }
$$

Since $\rho^{2} \neq 1$ and $r \neq 0, r\left(1+\rho^{2}\right)-2 \rho \cos (\theta-\varphi)=0$.

This equation expresses the N.E. line equidistant from 0 and $a_{0}^{\prime}$, and according as $\cos \theta-r\rangle,=,<0$ it intersects the radius $\overline{01}$ at only one inner point, at 1 , at no point respectively. These correspond to the cases: $a_{0}^{\prime}$ lies inside, upon, outside $r$ respectively.

Using this lemma we can show

THEOREM 3.6. Let $z_{0}$ be a regular point on $\Gamma$. If centres $\left\{a_{n}\right\}$ are suitably chosen, $z_{0}$ is the boundary point of only one N.P.

Proof. Taking the proof of Theorem 3.5 into account, we see that it is sufficient to prove that none of the sides of the N.P., having $z_{0}$ as its boundary point, ends at $z_{0}$, if centres are suitably chosen. According to Theorem 3.5 there exists a vicinity $V$ of $z_{0}$ in which $f(z)$ is univalent. Let $a_{0}$ be a point in $V$ and $\gamma$ be the inscribed circle, which passes through $a_{0}$ and touches $\Gamma$ at $z_{0}$. In view of Lemma 3.3 the circular arcs equidistant from $a_{0}$ and its equivalents

69) Cf. L. Bieberbach [1], 1 Aufl., pp. 57-58. 
$\left\{a_{n}\right\}$ do not intersect the arc $\widehat{a_{0} z_{0}}$. Therefore $\widehat{a_{0} z_{0}}$ belongs to the N.P. with centre $a_{0}$, and the sides of this N.P. do not end at $z_{0}$. Thus the proof is completed.

By Theorem 3.5 all the regular points on $\Gamma$ form an open set. We shall call each component a regular arc.

\section{Ideal boundary components ${ }^{i 01}$}

Let $\left\{\Re_{n}\right\}$ be a sequence of non-compact subsurfaces of $\Re$ such that $\Re_{1} \supset \Re_{2}$ $\supset \ldots, \bigcap_{n} \Re_{n}=\phi$ and the boundary, relative to $\Re$, of $\Re_{n}$ is a simple closed curve on $\mathfrak{R}$. Two such sequences $\left\{\mathfrak{R}_{n}\right\}$ and $\left\{\mathfrak{R}_{n}^{\prime}\right\}$ are called equivalent if for given $m$ there exists $n$ such that $\Re_{m} \supset \Re_{n}^{\prime}$ and $\Re_{m}^{\prime} \supset \Re_{n} \cdot{ }^{11\rangle}$ To equivalent sequences an ideal boundary component is made to correspond. We shall denote the set of all the ideal boundary components by $\mathfrak{\aleph}_{\Re}$. A topology is introduced on $\mathfrak{R}+\mathfrak{V}_{\Re}$ by neighborhoods as follows::2)

Original neighborhoods are taken on $\Re$. For a domain $\Re_{n}$ of a sequence determining $P_{\mathfrak{C}} \in \mathfrak{C}_{\Re}, \bar{\Re}_{n}$ is defined by the set consisting of $\Re_{n}$ and all the ideal boundary components $\left\{Q_{\mathfrak{C}}\right\}$ such that a domain of a determining sequence of $Q_{\mathfrak{C}}$ is contained in $\Re_{n}$. $\left\{\hat{\mathcal{R}}_{n}\right\}$ are taken as neighborhoods of $P_{\mathfrak{C}}$.

An ideal boundary component is said to belong to the first class if a domain of its determining sequence is of planar character. Otherwise it belongs to the second class. If $\Re_{n}$ is a surface of planar character of a determining sequence of $P_{\mathfrak{C}} \in \mathfrak{C}_{\Re}$ of the first class, it is mapped by Koebe's theorem ${ }^{33)}$ conformally onto a plane domain $D$. If $P_{\text {厄 }}$ corresponds to an isolated boundary point of $\dot{D}$, we will call $P_{5}$ parabolic, and any non-parabolic ideal boundary component, regardless of its class, hyperbolic. These definitions do not depend upon the choice of $\Re_{n}$ and the way of its mapping.

\section{Parabolic fixed points}

Similarly as in the case when $\Re$ is a plane domain, we can show that the cluster set $S_{z_{0}}^{(U)}$ coincides with $\Re$ at every singular point $z_{0}$. Clearly fixed points and their limiting points are singular, and conversely the singular points consist only of them. We shall investigate the parabolic fixed points in this section.

Let $z_{0}$ be a parabolic fixed point on $\Gamma$ and $\mathbb{B}_{0}$ be the subgroup of $\mathbb{B}$ consisting of all the parabolic substitutions having $z_{0}$ as their common fixed point.

70) Cf. B.v. Kerékjártó [1], Abschn. V, § 1, S. Stoïlow [1], pp. 85-92. They have called them Randstücke and éléments-frontières respectively, but here the terminology above is used to avoid the confusion with boundary elements, which will be defined in Chap. V.

i) As is shown in S. Stoillow [1], one induces the other of these inequalities.

:2) It can be shown that $\Re+\mathfrak{E}_{\Re}$ is a compactum and $\mathfrak{E}_{\Re}$ is totally-disconnected.

73) P. Koebe [1]. 
Since $\mathscr{G}_{0}$ is properly discontinuous in $U$, it is a cyclic group generated by a substitution $T_{0}$; that is, $\mathbb{G}_{0}=\left\{T_{0}^{n}\right\}(n=0, \pm 1, \pm 2, \ldots)$.

First we give

ThForem 3.7. Let $z_{0}$ be a parabolic fixed toint and divide $U$ into N.P.s in an arbitrary way. If an inscribed circle touching $\Gamma$ at $z_{0}$ is drawn sufficiently small, its inside is divided into a finite number of wedge-shaped parts of N.P.s.

Proof. ${ }^{-4)}$ Let $\gamma$ be an inscribed circle touching $\Gamma$ at $z_{0}$. By the diameter of $\gamma$ and its equivalents with respect to $\left\{T_{0}^{n}\right\}$, the inside of $\gamma$ is divided into wedges $\left\{W_{n}\right\}(n=0, \pm 1, \pm 2, \ldots)$.

Any N.P. lies between the two N.E. lines, which are equidistant from its centre $a_{0}$ and $T_{0}\left(a_{0}\right)$ or $T_{0}^{-1}\left(a_{0}\right)$ and terminate at $z_{0}$. Hence if an infinite sequence of N.P.s $\left\{N_{k}\right\}$ has common points with $W_{0}$, it tends to $z_{0}$ within $W_{1}+W_{0}+W_{-1}$. Therefore an infinite subsequence $\left\{N_{k(i)}\right\}$ exists such that their centres $\left\{a_{k(i)}\right\}$ lie in $W_{0}$ and every $N_{k(i)}$ has $z_{0}$ as its sole boundary point on $\Gamma$. The substitution which transforms $a_{k(1)}$ to $a_{k(2)}$ leaves $z_{0}$ invariant and so is parabolic. This contradicts the fact that $T_{0}$ is the generating one. Consequently only a finite number of N.P.s has common points with $W_{0}$. Hence if an inscribed circle is drawn sufficiently small, its inside is divided into a finite number of wedge-shaped parts of N.P.s.

Now we can prove the following theorem which is well-known when $\lambda^{2}$ is a plane domain ::5)

THEOREM 3.8. The classes of the equivalent parabolic fixed points on $\Gamma$ and the parabolic ideal boundary components of $\mathfrak{R}$ correspond to each other in a oneto-one manner.

Proof. Similarly as in the plane it can be shown that to a parabolic ideal boundary component there correspond equivalent parabolic fixed points on $\Gamma$.

The converse needs somewhat careful considerations. Divide $U$ into N.P.s in an arbitrary way. If an inscribed circle touching $T$ at a parabolic fixed point $z_{0}$ is drawn sufficiently small, a wedge $W_{0}$ defined by $T_{0}$ is divided into a finite number of smaller wedge-shaped parts $\left\{W^{(k)}\right\}(k=1, \ldots, p)$ of N.P.s by Theorem 3.7. Suppose that, however small an inscribed circle $\gamma$ may be taken, $f(z)$ were not univalent in $W_{0}$ inside $\gamma$. Then there would exist a substitution which transforms $W^{(i)}$ to $W^{(j)}(i \neq j)$ and leaves $z_{\text {j }}$ fixed. This contradicts the generating property of $T_{0}$. Hence $f(z)$ is univalent in $W_{0}$ inside a small $r$.

74) We make use of the ideas in L. Bieberbach [1], 1 Auf., pp. 59-60.

75) Cf. L. Bieberbach [1], 1 Auf.. pp. $30-31$ and 60-61, G. Julia [1], pp. $31-34$, for instance. 
In the second place map $U$ conformally onto the left half of the $W$-plane by $W(z)$ such that $z_{0}$ corresponds to $W=\infty$. Then to $T_{0}$ there corresponds a translation parallel to the imaginary axis and with the breadth $2 \pi \lambda$. Consider the function $Z=\exp (W / \lambda)$ and compose the mappings $Z \rightarrow W \rightarrow z \rightarrow \Re$. The corresponding function $P=\varphi(Z)$ is one-valued univalent in a sufficientily small $D: 0$ $<|Z|<r_{0}(<1)$, Consequently $D$ is conformally equivalent to a subsurface of planar character of $\Re$ and hence a parabolic ideal boundary component of $\mathfrak{R}$ corresponds to $Z=0$. Thus the proof is completed.

Moreover we can see that the generating $T_{0}$ corresponds to one rotation about the corresponding parabolic ideal boundary component.

Further, corresponding to Theorem 3.6 there holds

Theorem 3.9. Let $z_{0}$ be a parabolic fixed point on $\Gamma$. Then a division of $U$ into N.P.s and an inscribed circle $\gamma$ touching $I$ at $z_{0}$ can be chosen such that inside of $\gamma$ is divided into wedge-shaped parts of N.P.s and these parts are equivalent with respect to the generating parabolic substitution $T_{0}$.

Proof. As shown in the preceding proof, $f(z)$ is univalent in a wedge $W_{0}$ defined by $T_{0}$ inside a small circle touching $T$ at $z_{0}$. Take a point $a_{0}$ in $W_{0}$ and divide $U$ into N.P.s having $a_{0}$ and its equivalents as centres. The N.P. with the centre $a_{0}$ will be denoted by $N_{0}$, and the circle touching $\Gamma$ at $z_{0}$ and passing through $a_{0}$ by $\tau_{0}$. Since $f(z)$ is univalent in $W_{0}$, there is no equivalent of $a_{0}$ inside $\gamma_{0}$. Hence by Lemma $3.3{\widehat{a_{0} z_{0}}}_{0}$ is contained in $N_{0}$, and by Theorem 3.7 there exitst two sides of $N_{0}$ which terminate at $z_{0}$. According to Lemma 3.3 again these sides are equidistant from $a_{0}$ and its two nearest equivalents on $\gamma_{0}: T_{0}\left(a_{0}\right)$ and $T_{0}^{-1}\left(a_{0}\right)$. Hence the two sides are equivalent to each other with respect to $T_{0}$. Thus an inscribed circle sufficiently small is the required one.

Theorem 3.7 teaches us that only a finite number of the curves on $\Re$, which are the images of the sides of N.P.s, terminates at the parabolic ideal boundary component corresponding to $z_{0}$, and Theorem 3.9 that if a point $P_{0} \in \Re$ is taken sufficiently near the component, and if the images of $P_{0}$ in $U$ are taken as centres, a sole image of the sides terminates at the component.

The parabolic fixed points are dense in itself on $\Gamma$, if $\Re$ has at least one parabolic ideal boundary component, excepting the case when $\mathfrak{R}$ is doublyconnected.

\section{Hyperbolic fixed points}

We shall call a regular arc, whose end points are fixed points, completely regular. Except in the case when $\Re$ is a doubly-connected surface with a parabolic and a hyperbolic ideal boundary components, two end points of every com- 
pletely regular arc are fixed points of a hyperbolic substitution.

Corresponding to Theorem 3.8, we can show

THEOREM 3.10. The classes of the equivalent completely regular arcs and the isolated hyperbolic ideal boundary components of $\mathfrak{R}$ correspond to each other in a one-to-one manner.

Except in the cases when $\mathfrak{R}$ is of genus zero and its ideal boundary components consist of three parabolic, or of one parabolic plus one hyperbolic ideal boundary components, there exists always a curve on $\Re$, which is homotopic to neither zero nor a curve surrounding a parabolic ideal boundary component. Therefore the Fuchsian group \& contains at least one hyperbolic substitution. Further, hyperbolic fixed points are dense in itself on $\Gamma$.

\section{Non-fixed singular points}

All the singular points on $\Gamma$ form a closed set $S$ and are dense in itself on $I$ except in the case when $\Re$ is a doubly-connected surface. Hence $S$ has a power of continuum. Since fixed points are enumerable, the power of non-fixed singular points is of continuum.

Every parabolic fixed point is a boundary point of some N.P.s but none of hyperbolic fixed points is so. Similarly as in a plane ${ }^{76)}$ we have

THEOREM 3.11. The following propositions are equivalent to each other:

1) the connectivity of $\Re$ is finite;

2) there appears no non-fixed singular point on the boundary of every N.P.;

3) the boundary of an N.P. consists of only a finite number of sides.

In the proof of this theorem use is made of Theorems 3.7,3.8 and 3.10 and the fact that if the Fuchsian group $B$ is generated by a finite number of substitutions the connectivity of $\Re$ is finite.

\section{Chapter IV. Dirichlet Problems}

1. Problem on an open compact set in $\mathfrak{R}$

In Chap. I we treated Dirichlet problem in the case when $\mathscr{D}$ is an open compact true subset in $\Re$. We shall continue it in this section, assuming that the transfinite kernel of $\mathfrak{R}-\mathscr{D}$ is non-empty on $\Re$.

If we can show that any continuous boundary function $\varphi$ is resolutive, the similar treatments as by M. Brelot in euclidean spaces become possible. Actually we shall proceed on this way.

First we will prove

76) Cf. L. Bieberbach [1], 1 Aufl., pp. 63-64. 
LEMMA 4.1. There exists in $\mathfrak{D}$ a positive harmonic function $U_{0}(P)$ such that $U_{0}(P) \rightarrow+\infty$ when $P$ tends to every irregular point on the boundary $C$ of $\mathfrak{D}$.

Proof. Since $C$ is compact in $\mathfrak{R}$, it is covered by a finite number of simplyconnected domains $\left\{N_{k}\right\}(k=1, \ldots, n)$ bounded by analytic curves on $\Re$. We may, and shall, suppose that the transfinite kernel of the part of $C$ on each $N_{k}^{a}$ is non-empty on $\mathfrak{R}$. The mapping function $t=f_{k}(P)$ of $N_{k}$ onto $\Delta:|t|<1$, is analytic univalent also in a domain $N_{k}^{\prime} \supset N_{k}^{a}$ by the principle of reflexion. We shall provide a positive function on the $t$-plane such that it is harmonic outside the image $C_{1}$ on $\Delta^{a}$ of $C$, even at $t=\infty$, and tends to $+\infty$ as $t$ tends to the image $E_{1}$ on $\Delta^{a}$ of the irregular points $E$ on $C$.

Since $E_{1}$ is of capacity zero and an $F_{0}$ set, ${ }^{77}$ there exists a distribution of a unit mass on $E_{1}$ such that the potential $u_{1}(t)$, induced by it, is $+\infty$ on $E_{1}$; Further, since $C_{1}$ is of positive capacity, there exists the equilibrium potential $u_{2}(t)$ induced by a unit mass on $C_{1} .^{\text {s) }}$ Then $u_{1}(t)-u_{2}(t)$ is bounded below near $C_{1}$, tends to $+\infty$ as $t$ tends to $E_{1}$, and is harmonic outside $C_{\mathrm{s}}$, even at $t=\infty$, whence

$$
\int_{|t|=1+\varepsilon} \frac{\partial\left(u_{1}-u_{2}\right)}{\partial \nu} d s=0
$$

where $\varepsilon>0$ is taken sufficiently small so that $|t| \leqq 1+\varepsilon$ is contained in $f_{k}\left(N_{k}^{\prime}\right)$ $=\left\{f_{\bar{k}}(P) ; P \in N_{k}{ }^{\prime}\right\}$. By adding a constant the required positive function in the $t$-plane is obtained.

Thus Theorem 2.1 or 2.2 can be applied according as the boundary of $\Re$ is positive or null, and we obtain a positive function, which is harmonic on $i$ outside $N_{k}^{a} \cap C$ and tends to $+\infty$ as $P$ tends to $N_{k} \cap E$. The sum of such functions for all $\left\{N_{k}\right\}$ gives the function required in the theorem.

Now let $c$ be a continuous function on $C$. By (1.1) and Lemma 4.1 $\bar{H}_{p}^{\mathfrak{D}}(P)-\varepsilon U_{0}(P)$ belongs to $\mathfrak{U}_{p}^{\mathfrak{D}}$ for any $\varepsilon>0$. Hence $\bar{H}_{\varphi}^{\mathfrak{D}}(P)-\varepsilon U_{0}(P) \leqq \underline{H}_{\varphi}^{\mathfrak{D}}(P)$. $\varepsilon$ being arbitrarily small, there follows $\bar{H}_{P}^{\mathscr{D}}(P) \leqq \underline{H}_{\varphi}^{\mathscr{D}}(P)$, whence the equality. Since $H_{P}^{D}(P)$ is bounded clearly, $\varphi$ is resolutive. Further it can be shown that Wiener's solution exists and coincides with $H_{\varphi}^{\mathfrak{P}}(P) .^{: 9)}$

Since $H_{\uparrow}^{\mathfrak{D}}(P)$ is a linear functional of continuous $\varphi$, it is expressible by $\int_{C} \varphi d \mu^{p}$, where $\mu^{P}$ is a measure defined on the Borel class on $C$ and equal to the harmonic measure. For any $\varphi$ define lower and upper integrals by

77) Cf. M. Brelot [1].

:8) Cf. O. Frostman [1].

79) Cf. M. Brelot [2]. 


$$
\int_{C} \varphi d \mu^{P}=\sup \left\{\int_{C} \psi d \mu^{P} ; \phi \leqq \varphi \text { and } \psi \text { is upper bounded } \mu^{P} \text {-measurable }\right\}
$$

and

$$
\int_{C} \varphi d \mu^{P}=\inf \left\{\int_{C} \phi d \mu^{P} ; \psi \supseteqq \varphi \text { and } \psi \text { is lower bounded } \mu^{P} \text {-measurable }\right\}
$$

respectively, where $\pm \infty$ are granted to $\psi$. Then after M. Brelot ${ }^{801}$ we can show that

$$
\underline{H}_{\mathscr{P}}(P)=\underline{\int}_{C} \varphi d \mu^{P} \text { and } \bar{H}_{\mathscr{p}}^{\mathfrak{D}}(P)=\bar{\int}_{C} \varphi d \mu^{P} \text {. }
$$

If the class of $\mu^{P}$-measurable sets are defined as usual, it follows from these relations that in order that $\varphi$ is resolutive it is necessary and sufficient that $\varphi$ is $\mu^{P}$-integrable in the narrow sense.

\section{Problem on $\mathfrak{R}$ as a covering surface}

Let $\mathfrak{R}$ be an inner covering surface ${ }^{\text {\$1) }}$ with relative boundary ${ }^{\text {(2) }}$ over another Riemann surface. Then there exists at least one accessible boundary point of $\mathfrak{R}$. Further suppose that $\mathfrak{R}$ has a positive boundary. We shall consider Dirichlet problem on the complete metric space $\mathfrak{R}+\mathfrak{I}_{\Re}$ hereafter in this chapter, where $\mathfrak{I}_{\mathfrak{M}}$ represents the set of all the accessible boundary points of $\mathfrak{R} .^{{ }^{11}}$ )

Similarly as in euclidean spaces lower and upper classes $\mathfrak{H}_{\varphi}^{\Re}$ and $\mathfrak{B}_{\varphi}^{\Re}$, and hypo and hyper functions $\underline{H}_{\varphi}^{\Re}(P)$ and $\bar{H}_{\varphi}^{\Re}(P)$ are defined for any real function $\varphi$ on $I_{\Re}$. If there holds $u(P) \leqq 0$ on $\Re$ whenever $u(P)$ is upper bounded continuous subharmonic on $\Re$ such that $\varlimsup_{P \rightarrow T^{\prime} \in \mathbb{T}_{\Re}} u(P) \leqq 0$, we will call $\Re$ a surface of $D$-type. On $\Re$ of $D$-type there holds $\underline{H}_{\varphi}^{\Re}(P) \leqq \bar{H}_{\varphi}^{\Re}(P)$.

Since at present we are not able to show the resolutivity of continuous $\varphi$ generally, we shall consider the special case when $\Re$ is of finite connectivity and fulfills the condition $(\mathrm{C})^{\text {si) }}$ henceforth in this chapter.

Make $\mathfrak{R}$ into $\Re_{0}$ of planar character by $p$ disjoint simple closed analytic curves $\left\{\gamma_{i}\right\} \quad(i=1, \ldots, p)$. Since the number $2 p+n$, where $n$ is the number of the ideal boundary components of the first class of $\Re$, is equal to the connectivity of $\Re, n$ and $p$ are finite. Hence $\mathfrak{R}_{0}$ can be mapped onto a domain outside $2 p+n$ circles or points in the $\zeta$-plane. By the identification of each of $\not$ pairs of corresponding circles, we obtain a Riemann surface $\mathfrak{A}_{\zeta}$ to which $\Re$ is conformally equivalent. If each pair is identified for the plane domain bounded only by above $2 p$ circles, a closed Riemann surface $\Re_{\zeta}$ is obtained and this includes $\Re_{\zeta}$ as its true subsurface. The boundary $C_{\zeta}$ of $\Re_{\zeta}$ corresponds to $n$

80) M. Brelot [2], [5]. In euclidean spaces he expressed $H$ and $\bar{H}$ by Daniell integrals.

81) Cf. Chap. III, §1.

32) This means that $\Re$ is begrenzt in Weyl's sense. Cf. H. Weyl [1], p. 47. 
circles or points in the $\zeta$-plane, where at least one circle exists because $\Re$ has a positive boundary.

Further map the universal covering surface $\Re_{\zeta}^{\infty}$ conformally onto $U:|z|$ $<1$. Then to the boundary circles $C$ of $\Re_{\zeta}$ there correspond completely regular arcs on $I: z:=1$. Taking Theorems 3.1 and 3.2 into account in the mapping $\mathfrak{N}\left(\rightarrow \mathfrak{R}_{\zeta}\right) \rightarrow U$, we see that $\mathfrak{I}_{\mathfrak{R}}$ is mapped in a one-to-one continuous manner onto a set $E_{\zeta}$ on $C_{\zeta}$ and the image on $\Re$ of any curve terminating at a point on $E_{\zeta} \cap C$ but not touching $C$ determines a point of $\mathfrak{I}_{\Re} \cdot{ }^{33)}$ Hence the function $\varphi$ on $\mathfrak{T}_{\Re}$ is transformed into a function on $E_{\zeta}$, which will be denoted by the same letter $\xi$.

Since the transfinite kernel of $\Re_{\zeta}-\Re_{\zeta}$ is non-empty on $\Re_{\zeta}$, Dirichlet problem is manageable on $\mathfrak{R}_{\zeta}$ by the procedure in the preceding section.

First we will prove

THEOREM 4.1. Let $\mathfrak{R}$ be a Riemann surface stated above and of D-type, and $\varphi$ a bounded Borel function on $\mathfrak{I}_{\Re}$. Then $\varphi$ is resolutive, and if it is extended to a function on $C_{\zeta}$ by an arbitrary way, there holds $H_{\varphi}^{\Re}(P(\zeta))=H_{\Phi}^{\Re} \zeta(\zeta)$.

Proof. First we fix a point $\zeta_{0}$ on $\Re_{\zeta}$. Since the mapping of the complete metric space $\mathfrak{I}_{\Re}$ onto $E_{\zeta}$ is one-to-one continuous, the image of a Borel set on $\mathfrak{I}_{\mathfrak{R}}$ is a Borel set on $E_{\zeta}$. Therefore the function $\bar{\Phi}$ on $C_{\zeta}$, which is the extension of $\varphi$ by a constant $\bar{M}>\sup _{E_{\zeta}} \varphi$, is $\mu^{\zeta_{0}}$-measurable, where $\mu^{\zeta_{0}}$ is the harmonic measure on $\mathfrak{A}_{\zeta}$ at $\xi_{0}$. On account of Lusin's theorem, we can find for any integer $n$ an open set $G_{n} \subset C_{\zeta}$ such that $\mu^{\zeta_{0}}\left(G_{n}\right)<1 / n$ and $\bar{\Phi}$ is continuous on $C_{\zeta}-G_{n}$ $=F_{n}$. A function $\Phi_{n}$ defined by $\bar{\Phi}$ on $F_{n}$ and by $\underline{M}=\inf _{E_{\zeta}} \varphi$ on $G_{n}$ is upper semicontinuous. Hence there holds $\varlimsup_{\zeta \rightarrow \zeta^{\prime}} H_{\Phi_{n}}^{\Re \zeta}(\zeta) \leqq \Phi_{n}\left(\zeta^{\prime}\right)$ on $C^{E \zeta}$ by (1.1). Especially on $E_{\zeta} \cap C$

$$
\varlimsup_{\zeta \rightarrow \zeta^{\prime}} H_{\Phi_{n}}^{\Re \zeta}(\zeta) \leqq \Phi_{n}\left(\zeta^{\prime}\right) \leqq \bar{\Phi}\left(\zeta^{\prime}\right)=\varphi\left(\zeta^{\prime}\right)
$$

According to Lemma 4.1 there exists on $\mathfrak{R}_{\zeta}$ a positive harmonic function $U_{0}(\zeta)$ such that $U_{i 1}(\xi) \rightarrow+\infty$ when $\zeta$ tends to every isolated point of $C_{\zeta}$. Consequently, if $H_{\Phi_{n}}^{\Re \zeta}(\zeta)-\varepsilon U_{n}(\zeta)$ for any $\varepsilon>0$ is regarded as a function on $\Re$, it belongs to the lower class $\mathfrak{U}_{\hat{r}}^{\Re}$ and hence $H_{\Phi_{n}}^{\Re \zeta}(\zeta)-\varepsilon U_{0}(\zeta) \leqq \underline{H}_{\phi}^{\Re}(P(\zeta))$. $\varepsilon$ being arbitrarily small, there follows $H_{\Phi=}^{\Re \zeta}(\zeta) \leqq \underline{H}_{p}^{\Re}(P(\zeta))$. Further there holds

$$
0 \leqq H_{\Phi}^{\Re}\left(\zeta_{0}\right)-H_{\Phi_{n}}^{\Re_{\zeta}}\left(\zeta_{0}\right) \leqq \int_{G_{n}}\left(\Phi-\Phi_{n}\right) d \mu^{\zeta_{0}} \leqq(\bar{M}-\underline{M}) \mu^{\zeta_{0}}\left(G_{n}\right)<\frac{1}{\mathrm{n}}(\bar{M}-\underline{M}) .
$$

8:. If these facts are known to us in some way, it is needless to impose the condition

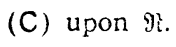


When $n \rightarrow \infty$ the last term tends to zero and follows

$$
H_{\bar{\Phi}}^{\Re \zeta}\left(\zeta_{0}\right)=\lim _{n \rightarrow \infty} H_{\Phi_{n}}^{\Re \zeta}\left(\zeta_{0}\right) \leqq \underline{H}_{\varphi}^{\Re}\left(P\left(\zeta_{0}\right)\right) .
$$

If we define $\Phi$ by extending $\varphi$ by $\underline{M}$, we obtain

$$
\bar{H}_{\varphi}^{\Re}\left(P\left(\zeta_{0}\right)\right) \leqq H_{\Phi}^{\Re \zeta}\left(\zeta_{0}\right) \leqq H_{\Phi}^{\Re \zeta}\left(\zeta_{0}\right)
$$

in a similar way. Since $\underline{H}_{\varphi}^{\Re}(P) \leqq \bar{H}_{\varphi}^{\Re}(P)$, there holds

$$
\underline{H}_{\varphi}^{\Re}\left(P\left(\zeta_{0}\right)\right)=\bar{H}_{\varphi}^{\Re}\left(P\left(\zeta_{0}\right)\right)=H_{\bar{\Phi}}^{\Re \zeta}\left(\zeta_{0}\right)=H_{\Phi}^{\Re \zeta}\left(\zeta_{0}\right) .
$$

$\zeta_{0}$ being arbitrary, $\varphi$ is resolutive and on $\Re_{\zeta}$

$$
H_{\varphi}^{\Re}(P(\zeta))=H_{\Phi}^{\Re \zeta}(\zeta)=H_{\Phi}^{\Re \zeta}(\zeta)
$$

In this we see that the harmonic measure of $C_{\zeta}-E_{\zeta}$ on $\Re_{\zeta}$ is zero and hence the way of extension of $\varphi$ is arbitrary.

We shall use the same letter $\varphi$ to represent the function on $C_{\zeta}$, which is an extension of $\varphi$ defined on $E_{\zeta}$.

This theorem shows that $\Re$ of D-type is of F-type ${ }^{84)}$ under our conditions. The converse is given by

THEOREM 4.2. If $\Re$ is of F-type, then it is of D-type. ${ }^{85)}$

Proof. Let $u(P)$ be an upper bounded continuous subharmonic function on $\Re$ such that $\varlimsup \lim u(P) \leqq 0$ as $P \rightarrow P^{\prime} \in \mathfrak{T}_{\Re}$. We shall denote the function $u(P(z))$ in $U$ by $\bar{u}(z)$, and the image on $\Gamma$ of $\mathfrak{I}_{\Re}$ by $E_{z}$. Along any radius terminating at a point of $E_{z}$, there holds $\overline{\lim } \bar{u}(z) \leqq 0$.

Now fixing any point $z_{0}$ we shall show $\bar{u}\left(z_{0}\right) \leqq 0$. Without loss of generality we may suppose $z_{0}=0$. By Egoroff's theorem we can find for any integer $n$ a closed set $F_{n} \subset E_{z}$ such that the linear measure $m\left(\Gamma-F_{n}\right)<1 / n$ and $M_{r}=\sup \left\{\bar{u}\left(r e^{i \theta}\right)\right.$; $\left.\epsilon^{i n} \in F_{n}\right\}$ tends to a non-positive value as $r \rightarrow 1$. For a function $\varphi_{r}$ defined on $|z|=r$ by $\bar{u}\left(r e^{i \theta}\right)$ for $e^{i \theta} \in F_{n}$ and by $\bar{M}=\sup _{\Re} u$ for other $z$, there holds

$$
\bar{u}(0) \leqq \frac{1}{2 \pi} \int_{0}^{2 \pi} \varphi_{r}\left(r e^{i \theta}\right) d \theta \leqq \frac{\bar{M} \cdot m\left(\Gamma-F_{n}\right)}{2 \pi}+M_{r}<\frac{\bar{M}}{2 \pi n}+M_{r} .
$$

Since as $r \rightarrow 1 \lim M_{r} \leqq 0$ and $n$ may be taken arbitrarily large, $\bar{u}(0) \leqq 0$. Thus the proof is completed.

Consequently if $\mathfrak{R}$ does not cover a set having a non-empty kernel on the

84) See Chap. III, § 2.

85) We impose no other condition upon $\Re$ here. 
basic surface, $\Re$ is of $D$-type by Theorem 3.3. Hereafter in this chapter we shall suppose that $\Re$ is of $D$-type.

If for a fixed $P_{0} \in \mathfrak{R}$ a set function $\mu^{P}(B)$ on the Borel class $\mathfrak{B}$ on $\mathfrak{I}_{\mathfrak{R}}$ is defined by $H_{\chi_{B}}^{\Re}(P)$, where $\gamma_{B}$ is the characteristic function of $B$, it is a measure on $B$ on account of Theorem 4.1. Hence we can define lower and upper integrals for any function, and $\mu^{P}$-measurability of sets on $\mathfrak{I}_{\Re}$.

The following two lemmas are given to represent $\underline{H}_{\varphi}^{\Re}(P)$ and $\bar{H}_{\varphi}^{\Re}(P)$ by integrals :

LEMMA 4.2.86) $\underline{H}_{7}^{\Re}(P)$ is the upper cover of $\underline{H}_{\psi}^{\Re}(P)$, where $\psi \leqq \varphi$ and $\psi$ is upper bounded and upper semicontinuous on $\mathfrak{I}_{\Re}$. The similar fact holds for $\bar{H}_{\varphi}^{\Re}(P)$.

LEMMA 4.3. If $\varphi$ is an upper bounded Borel function on $\mathfrak{I}_{\Re}$, then

$$
\underline{H}_{\varphi}^{\Re}(P)=\bar{H}_{\vartheta}^{\Re}(P)=\int_{\mathfrak{T}_{9}} \varphi d_{\mu}{ }^{P}
$$

on $\Re$.

Proof. It is sufficient to prove this for $\varphi \leqq 0$, because $\underline{H}_{\varphi_{-}}^{\Re}(P)=\underline{H}_{\varphi}^{\Re}(P)-M$ and $\bar{H}_{p-M}^{\Re}(P)=\bar{H}_{\varphi}^{\Re}(P)-M$ for any finite constant $M$. Hence $\varphi$ will be supposed to be non-positive.

When $\varphi$ is bounded, it can be shown without difficulty that $H_{\varphi}^{\Re}(P)=\int_{\mathfrak{T}_{\mathfrak{R}}} \varphi d \mu^{P}$. For any Borel $\varphi \leqq 0$ we have by Lemma 4.2 and Vitali-Carathéodory's theorem ${ }^{\mathrm{s} i}$

$$
\bar{H}_{\varphi}^{\Re}(P)=\inf _{\psi} H_{\psi}^{\Re}(P)=\inf _{\psi} \int_{\mathfrak{D}_{\Re}} \psi d \mu^{P}=\int_{\mathfrak{T}_{\mathfrak{R}}} \varphi d \mu^{P},
$$

where $\psi \supseteqq \varphi$ and $\psi$ is bounded lower semicontinuous on $\mathfrak{I}_{\mathfrak{R}}$. If $\int_{\mathfrak{T}_{\mathfrak{R}}} \varphi d \mu^{\mu}=-\infty$ there holds $\underline{H}_{p}^{\Re}(P) \equiv \bar{H}_{\varphi}^{\Re}(P) \equiv-\infty$. Hence suppose $\int_{\mathbb{L}_{\Re}} \varphi d_{\mu} l^{P}>-\infty$.

Now divide $[0,-\infty)$ such that $0=c_{0}>c_{1}>c_{2}>\ldots$ and denote the set $\{P$; $c_{n}\left\langle\varphi(P) \leqq c_{n-1}\right\rangle$ dy $\mathfrak{E}_{n}$. Then $\int_{\mathfrak{L}_{\mathfrak{n}}} \varphi \mathrm{d} \mu^{P}=\sum_{n} \int_{\mathfrak{E}_{n}} \varphi d \mu^{P}=\sum_{n} H_{p_{n}}^{\Re}(P)$, where $\varphi_{n}=\varphi$ on $\mathfrak{F}_{n}$ and $=0$ on $\mathfrak{I}_{\Re}-\mathfrak{F}_{n}$. Let $u_{n}(P)$ be a continuous subharmonic function such that given $\varepsilon>0$ and a fixed point $P_{0}, H_{\varphi_{n}}^{\Re}\left(P_{0}\right)<u_{n}\left(P_{0}\right)+\frac{\varepsilon}{2^{n}}$ and $\overline{\lim } u_{n}(P)$

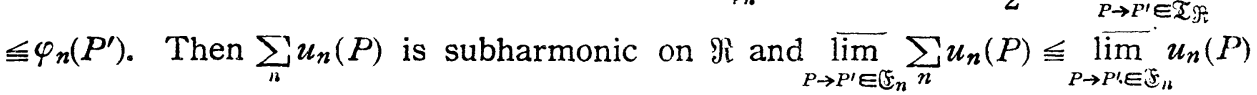
$\leqq \varphi_{n}\left(P^{\prime}\right)=\varphi\left(P^{\prime}\right)$. This shows that $\sum_{n} u_{n}(P) \in \mathfrak{U}_{2}^{\Re}{ }^{98)}$ Therefore $\sum_{n} H_{p_{n}}^{\Re}\left(P_{0}\right)$

86) Cf. M. Brelot [2]. He proved this lemma in euclidean spaces.

8:) Cf. S. Saks [1], pp. 75-76.

88) If $\sum_{n} u_{n}(P)$ is not continuous, it is necessary, and possible, to replace it by the greater continuous one which belongs to $\mathfrak{u}_{\varphi} \mathfrak{p}$. Cf. M. Brelot [2] 
$\lesssim \sum_{n} u_{n}\left(P_{0}\right)+\varepsilon \leqq \underline{H}_{\rightarrow}^{\Re}\left(P_{0}\right)+\Xi . \quad$ being arbitrarily small, $\sum_{n} H_{p_{n}}^{\Re}\left(P_{v}\right) \leqq \underline{H}_{\varphi}^{\Re}\left(P_{0}\right)$. Since this holds for any $P_{v} \in \mathfrak{\Re}$, we have $\sum_{n} H_{\not n}^{\Re}(P) \leqq \underline{H}_{\supsetneq}^{\Re}(P)$. Therefore holds

$$
\bar{H}_{P}^{\Re}(P)=\int_{\mathfrak{T}_{\Re}} \varphi d \mu^{P}=\sum_{n} H_{\stackrel{\gamma}{\Re}}^{\Re}(P) \leqq \underline{H}_{P}^{\Re}(P) \leqq \bar{H}_{p}^{\Re}(P) .
$$

Hence there follows

$$
\underline{I I}_{\rho}^{\Re}(P)=\bar{H}{ }_{\varphi}^{\Re}(P)=\int_{\mathfrak{T}_{\Re}} \varphi d u^{P} .
$$

Taking Lemmas 4.2 and 4.3 into account we have at once

THEOREM 4.3. There hold for any

$$
\underline{H}_{P}^{\Re}(P)=\int_{\mathfrak{T}_{\Re}} \varphi d \mu^{P} \quad \text { and } \quad \widetilde{H}_{\phi}^{\Re}(P)=\int_{\mathfrak{T}_{\mathfrak{R}}} \varphi d \mu^{P} .
$$

Corollary. In order that $\varphi$ is resolutive it is necessary and sufficient that $\varphi$ is $\mu^{P}$-integrable in the narrow sense.

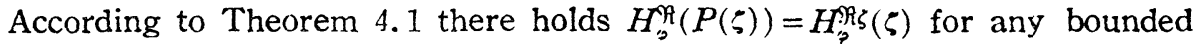
Borel function $\varphi$ on $\mathfrak{I}_{\mathfrak{R}}$. Any upper bounded Borel function $\varphi$ can be approximated by bounded Borel functions $\left\{\varphi_{n}\right\}$ such that $\varphi_{n} \downarrow \varphi$. Since $H_{\varphi_{n}}^{\Re}(P(\zeta))$ $=H_{\varphi_{n}}^{\Re \zeta(\zeta)}$ and we can express them by integrals, the limits may be taken and

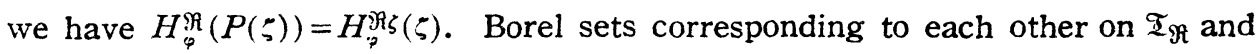
$C_{\zeta}$, we get further by Lemma 4.2

THEOREM 4.4. There hold for any

$$
\underline{H}_{p}^{\Re}(P(\zeta))=\underline{H}_{p}^{\Re \zeta(\zeta)} \text { and } \bar{H}_{p}^{\Re}(P(\xi))=\bar{H}_{\varphi}^{\Re \zeta}(\zeta) \text {. }
$$

Let $\mathfrak{R}^{\prime}$ be a covering surface of finite connectivity over $\mathfrak{R}$ which has been considered hitherto. Then the set $\mathfrak{I}_{\mathfrak{R}}$, of all the accessible boundary points of $\Re^{\prime}$ are defined relatively to the basic surface of $\Re$, and $\Re^{\prime}+\mathcal{T}_{\Re}$. may be considered as a kind of covering surface over $\mathfrak{i}+\mathfrak{I}_{\mathfrak{R}}$. Further we assume that $\mathfrak{R}^{\prime}$ is of D-type too. ${ }^{99}$ Under these conditions we can prove in a similar way as M. Brelot ${ }^{201}$

TheOREM 4.5. Let $\varphi(P)$ be a function $\mathfrak{T}_{\Re}$, and define a function $\psi\left(P^{\prime}\right)$ on $T_{\Re^{\prime}}$ by $\underline{H}_{\varphi}^{\Re}(P)$ or $\bar{H}_{, P}^{\Re}(P)$ when $P^{\prime}$ lies over an inner point $P$ of $\Re$, and by $\varphi(P)$ when $P^{\prime}$ lies over a point $P$ of $\mathfrak{I}_{\Re}$. Then there holds

$$
\underline{H}_{\psi}^{\Re^{\prime}}\left(P^{\prime}\right)=\underline{H}_{q}^{\Re}(P) \quad \text { or } \quad \bar{H}_{?}^{\Re \prime}\left(P^{\prime}\right)=\bar{H}_{\varphi}^{\Re}(P)
$$

39) We will propose a question whether $\Re^{\prime}$ over $\Re$ of $D$-type is always of D-type too or not necessarily so.

90) M. Brelot [5]. 
respectively.

Up to this place in this section we have investigated Dirichlet problem on $\mathfrak{R}$ of finite connectivity. But when $\mathfrak{A}$ is a compact domain $\mathfrak{D}$ in a Riemann surface and the transfinite kernel of its complement is non-empty, Dirichlet problem on $\mathfrak{I}+\mathfrak{I}_{D}$ can be solved in a similar way as by $\mathrm{M}$. Brelot in euclidean spaces, ${ }^{90)}$ even if the connectivity of $\mathfrak{T}$ is infinite.

On the other hand, R. Nevanlinna ${ }^{\text {:1 }}$ treated Dirichlet problem on $\mathcal{D}$ as follows :

Map $\mathfrak{D}^{*}$ onto $U:|z|<1$ conformally. Then by Theorem 3.3 $\mathfrak{I}_{\mathfrak{D}}$ is mapped to a set $E_{2}$ with measure $2 \pi$ on $\Gamma:|z|=1$, and a function $\varphi$ on $T_{\mathfrak{D}}$ is transformed into a function $\psi$ on $E_{z}$, where the function may be supposed to be defined on $\Gamma$. If the image on $\Gamma$ of a set $E \subset^{\mathfrak{I}_{\mathfrak{T}}}$ is linearly measurable on $\Gamma$, $E$ is called harmonically measurable, and if $\psi$ is integrable on $r$ the Poisson integral ${ }^{92}$ with the boundary function $\psi$ on $U$ is transformed into a one-valued harmonic function on $\mathfrak{T}$ and this is called the solution.

We can prove a theorem similar to Theorem 4.5: There hold $\underline{H}^{D^{\infty}}\left(P^{\prime}\right)$ $=\underline{H}_{\varphi}^{\mathscr{D}}(P)$ and $\bar{H}_{\psi}^{\mathfrak{T}^{\infty}}\left(P^{\prime}\right)=\bar{H}_{\varphi}^{\mathscr{D}}(P)$, where $\psi$ is the function on $\mathcal{T}_{D^{\infty}}$ defined by means of $\varphi$. From this and Theorem 4.4 we have

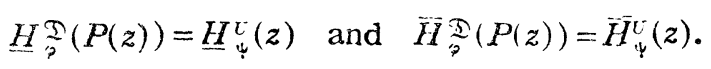

In these relations we see that Nevanlinna's method is equivalent to the method by Brelot and us.

Though our method was applied only for a compact domain in a Riemann surface or an inner covering surface of finite connectivity, Nevanlinna's method is available for any surface of $F$-type irrespective of its connectivity..$^{93}$ This gap is not yet filled at present.

\section{Applications}

Applications of Theorem 4.5 will be discussed in thie section.

Application 1. (Extension of Löuner's lemman ${ }^{(-1)}$ ). Let $w=f(z)$ be a bounded regular function in $U:|z|<1$ and suppose $f(0)=0,|f(z)|<1$. Denote by $f\left(e^{i \theta}\right)$ the limiting value of $f(z)$ at $z=e^{i \theta}$ along Stolz's paths, which exists a. $\mathrm{e}$

91) R. Nevanlinna [3]. He treated the case when $\%$ is defined on the ordinary boundary $C$ of $\mathscr{S}$. If $g$ is regarded as a boundary function on $T S$, the hypo and hyper functions obtained on $\mathcal{D}$ as on $\Re$ in this section coincide with those discussed in $\S 1$ of this chapter.

n2) For any integrable $\phi$ the Poisson integral equals $H_{p}^{U}$.

93) We can prove that any Borel set on $\mathfrak{T} 9$ is harmonically measurable.

94) K. Löwner [1]. Y. Kawakami [1]. S. Kametani and T. Ugaheri [1]. M. Tsuji [2]. 
on $T:|z|=1$ by Fatou's theorem. Taking a set $X$ on $T$ where $\left|f\left(e^{i \theta}\right)\right|=1$, the set $\left\{f\left(e^{i \theta}\right) ; e^{i \theta} \in X\right\}$ on the $w$-plane will be denoted by $X_{w}$.

Now we shall apply Theorem 4.5 , by considering $|w|<1$ as $\Re$, the Riemann surface of the inverse function of $f(z)$ as $\mathfrak{R}^{\prime}$ and the characteristic function of $X_{w}$ as the boundary function $\varphi$ on $\mathfrak{I}_{\Re}=\{|w|=1\}$. Then by Theorem 4.5

$$
\underline{m}\left(X_{w}\right)=\underline{H}_{\varphi}^{\Re}(0)=\underline{H}_{\psi}^{\Re^{\prime}}([0]) \supseteqq \underline{H}_{\psi_{1}}^{\Re^{\prime}}([0]),,^{95}
$$

where $\psi_{1}$ is a function on $\mathfrak{I}_{\Re}$ defined by 1 at points which correspond to points on $X$ and by 0 at other points and [0] is the point on $\Re^{\prime}$ corresponding to $z=0$.

By Theorem 4.4

and hence we have

$$
\underline{H}_{\psi_{1}}^{\Re^{\prime}}([0])=\underline{H}_{\psi_{1}}^{U}(0)=\underline{m}(X)
$$

Similarly there holds

$$
\begin{aligned}
& \underline{m}\left(X_{w}\right) \supseteqq \underline{m}(X) . \\
& \bar{m}\left(X_{w}\right) \supseteqq \bar{m}(X) .
\end{aligned}
$$

If $X$ is an analytic set on $\Gamma, X_{w}$ is so too and hence linearly measurable. Therefore we can write the above inequalities in $m\left(X_{w}\right) \triangleq m(X)$. However, even if $X$ is measurable on $\Gamma, X_{w}$ is not necessarily measurable on $\mathfrak{I}_{\Re} . \quad \mathrm{N}$. Lusin-J. Priwaloff and P. J. Myrberg ${ }^{96}$ ) have shown that there exist cases in which $m(X)$ $=0$ but $m\left(X_{w}\right)>0$. If a non-measurable subset $X_{w}^{\prime} \subset X_{w}$ is chosen, the set $\left\{z ; f(z) \in X_{w}^{\prime}\right\} \cap X=X^{\prime}$ is a null set on $\Gamma$. Thus $X^{\prime}$ is measurable but $X_{w}^{\prime}$ is not so. Conversely let $X_{w}$ be a measurable set, and put $\left\{z ; f(z) \in X_{w}\right\}=X$. Since by Theorems 4.4 and 4.5 there holds $H_{\varphi}^{\Re}(w)=H_{\psi}^{\Re \prime}\left(P^{\prime}\right)=H_{\psi}^{U}(z), \psi$ is measurable. Thus $X=\{z ; \psi(z)=1,|f(z)|=1\}$ is measurable.

Next we shall investigate the sign $\triangleq$. Taking account of Theorem 4.3 there holds

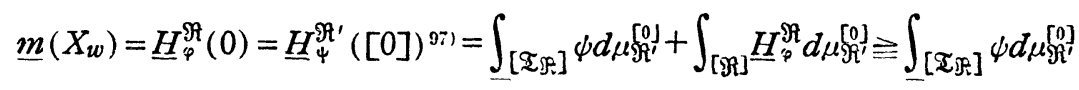

$$
\begin{aligned}
& \supseteqq \int_{\left[\mathfrak{Z} \Re_{2}\right]} \psi_{1} d \mu_{\mathfrak{R}^{\prime}}^{[0]}=\underline{m}(X),
\end{aligned}
$$

where $\left[\mathfrak{I}_{\Re}\right]$ and $[\Re]$ mean the subsets of $\mathfrak{I}_{\mathfrak{R}}$ whose projections lie on $\mathfrak{I}_{\mathfrak{R}}$ and $\Re$ respectively. If $\underline{m}\left(X_{w}\right)>0$, then $\underline{H}_{\varphi}^{\Re}(0)$ and hence $\underline{H}_{\varphi}^{\Re}(w)>0$. If $\mu_{\mathfrak{⿰}}^{[0]}([\Re])$ $=0$, then the harmonic measure of the points on $\Gamma$, whose image by $f(z)$ lies

95) $\underline{m}$ and $\bar{m}$ denote the Lebesgue linear inner and outer measure respectively. When $\underline{m}=\bar{m}$, it is denoted by $m$.

96) N. Lusin and J. Priwaloff [1]. P. J. Myrberg [2].

97) This equality holds, because $\underline{H}_{?}^{\Re}$ is harmonic and hence $\mu_{\Re^{\prime}}^{[0]}$-measuratle. 
in $\Re$, is zero by Theorem 4.4. This means that $f(z)$ belongs to class (U), ${ }^{39}$ ? and conversely if $f(z)$ belongs to class $(U)$ then $\mu_{\Re^{\prime}}^{[0]}([\Re])=0$. Now it has become clear that if $f(z) \notin$ class $(\mathrm{U})$ and $\underline{m}\left(X_{w}\right)>0$, then $\underline{m}\left(X_{w}\right)>\underline{m}(X)$. The same is true for $\bar{m}$. Moreover we can show that if $f(z) \in$ class (U) and $X=\left\{z ; f(z) \in X_{w}\right\}$, then $\underline{m}\left(X_{w}\right)=\underline{m}(X)$ and $\bar{m}\left(X_{w}\right)=\bar{m}(X)$.

These results include all the known results as the extension of Löwner's lemma.

Application 2. Let $f(z)$ be the same function as in the preceding application. If a harmonic function $u(w)$ can be expressed by a Poisson integral in $\Re:|w|<1$, the composed function $u(f(z))$ is also so in $U^{99}$ We shall prove this theorem using Theorem 4.5.

Let $\varphi$ be the boundary value of the Poisson integral of $u(w)$. Using the notation $\psi$ in Theorem 4.5, it follows by Theorems 4.4 and 4.5 that

$$
u(w)=H_{\varphi}^{\Re}(w)=H_{\psi}^{\Re \prime^{\prime}}\left(P^{\prime}\right)=H_{\psi}^{U}(z) .
$$

Hence $u(f(z))=H_{\psi}^{U}(z)$. This shows that $u(f(z))$ can be expressed by the Poisson integral with the boundary function $\psi$.

\section{Regular points}

We shall call $P_{0} \in \mathfrak{T}_{\Re}$ a regular point, if $\lim _{P \rightarrow p_{0}} H_{\varphi}^{\Re}(P)=\varphi\left(P_{0}\right)$ for any bounded function $\varphi$ on $\mathfrak{I}_{\Re}$ continuous at $P_{0}$. Otherwise the point will be called irregular. At a regular point $P_{0}(1.1)$ and $\left(1.1^{\prime}\right)$ hold good. Further, if $\lim _{P \rightarrow P_{0}} u(P)=\lambda<+\infty$ for a subharmonic function $u(P)$ and if the inner harmonic measure of a set $E \subset \mathfrak{I}_{\Re}$ is zero, then $P_{0}$ is an accumulating point of $\mathfrak{I}_{\Re}-E$ and there holds

$$
\lim _{P_{0} \neq P^{\prime} \rightarrow P_{0}}\left(\varlimsup_{P \rightarrow P^{\prime} \in \mathfrak{T}_{\mathbb{R}-E}} u(P)\right)=\lambda .^{100)}
$$

Therefore at regular points a principal theorem of cluster sets for analytic functions $^{101)}$ is valid.

At irregular points, however, it holds not necessarily, differing from the case in euclidean spaces. We shall show an example for it.

Let $\Re$ be the part, over $|w|<1$, of the Riemann surface of the function $\log w$, and map $\mathfrak{R}$ onto $U:|z|<1$. Then $w=0$ corresponds to a point $z_{0}$ on $I$ : $|z|=1$. When $\Re$ represents the part of $\Re$ which corresponds to a semi-circular disc of $U$ divided by the diameter through $z_{0}$, the harmonic measure on $\Re$ of

\footnotetext{
99) Cf. W. Seidel [2] and O. Frostman [1].

99) Cf. M. Ohtsuka [1].

100) Cf. M. Brelot [3], [5]. He proved these results in euclidean spaces.

101) See Lemma 5.1.
} 
the part of its boundary over $|w|=1$ will be denoted by $u(P)$. This function tends to 0 as $P$ tends to the boundary point $P_{0}$ over $w=0$ along the boundary of $\Re$, but to positive values as $P$ tends to $P_{0}$ on paths, whose images in $U$ make angles between 0 and $\frac{\pi}{2}$ with $\Gamma$. Consequently if $\exp (u(P)+i v(P))$, where $v(P)$ is the conjugate function of $u(P)$, is considered on $\Re$, the theorem corresponding to Lemma 5.1 does not hold for it at $P_{0}$.

There exists the Green function on $\Re$, and it tends to 0 even at the irregular point $P_{0}$, differing from the case in euclidean spaces.

Next subject tasked to us is to estimate the measure of irregular points on $i_{\Re}$. At present, however, we have no knowledge about it.

\section{Chapter V. Conformal Mapping of a Riemann Surface With a Rectifiable Boundary}

\section{A lemma from the theory of cluster sets}

In the usual theory of cluster sets we investigate cluster sets of functions on a plane domain into another plane. If a Riemann surface $\mathfrak{R}$ is an inner covering surface ${ }^{102)}$ over a basic surface, and if $f(z)$ is an analytic function on $\because \pi$ into another Riemann surface, then cluster sets can be defined for $f(z)$ at any point of $\mathfrak{I}_{\Re}$. However, a case where a fundamental theorem (below Lemma 5.1) does not hold was shown in the end of Chap. IV. Therefore we shall consider functions on plane domains into Riemann surfaces. For these functions usual methods are available to some extent, and certain results are obtained. But merely a fact, which will be used later in this chapter, will be stated in the following.

Let $D$ be a plane domain, $C$ its boundary, $z_{0}$ a point on $C, \Re$ a Riemann surface, and $f(z)$ an analytic function on $D$ into $\mathfrak{R}$. We denote the part of $D$ and $C$ in $\left|z-z_{0}\right|<r$ by $D_{r}$ and $C_{r}$ respectively, and the set of values taken by $f(z)$ in $D_{r}$ by $\mathscr{S}_{r}$. Then $S_{z_{0}}^{(D)}$ is defined by $\bigcap_{r>0}\left(\mathfrak{D}_{r}\right)^{a}$, ${ }^{103)} S_{z_{0}}^{(C)}$ by $\bigcap_{r>0}\left(\bigcup_{z_{0} \neq z \in r_{r}} S_{z}^{(D)}\right)^{a}$.

Using these notations we have

LEMMA 5.1. (Extension of Iversen's theorem ${ }^{10-1)}$ ). Let $z_{0}$ be a non-isolated boundary point of $D$. Then there holds $\left(S_{z_{0}}^{(n)}\right)^{b} \subset\left(S_{z_{0}}^{(r)}\right)^{b}$, where $S^{b}$ denotes the boundary of $S$ taken relatively to $\Re$.

Proof. Suppose that there is a point $P_{0} \in \Re$ such that it belongs to $\left(S_{z_{u}}^{(D)}\right)^{b}$ but not to $\left(S_{z_{0}}^{(r)}\right)^{b}$. Then we can select a small number $r>0$ and a neighborhood 
$N$ of $P_{0}$ such that $N^{a} \cap\left(\bigcup_{z_{0} \neq z \in C_{r}} S_{z}^{(D)}\right)^{a}=\phi$. Let $|t|<1$ be a local parameter circle corresponding to $N$, in which $t=0$ is the image of $P_{0}$, and $\Delta$ the inverse image of $N$ by $f(z)$ in $D$. Then $z_{0}$ is a non-isolated boundary point of $\Delta$, and for the composed function $t(f(z))$ on $\Delta$ into the $t$-plane, the cluster set $S_{z_{0}}^{\left(\Delta^{b}\right)}$ lies on $|t|=1$. On the other hand $S_{z_{0}}^{(\Delta)}$ has $t=0$ as its boundary point. This contradicts the following theorem due to M. Brelot: ${ }^{105}$ )

If $D$ is a bounded open set in the z-plane, if $z_{0}$ is a non-isolated boundary point of $D$, and if $f(z)$ is regular bounded in $D$, then $\left(S_{z_{0}}^{(D)}\right)^{b} \subset\left(S_{z_{0}}^{\left(D^{b}\right)}\right)^{b}$.

\section{Measure-theoretic lemmas}

In the first place a theorem due to $\mathrm{H}$. Federer ${ }^{106)}$ will be cited as

LEMMA 5.2. If $P=f(p)$ is a function on a metric space $\Omega$ into another space $\sigma$, if $A$ is an outer measure on $\sigma$, if $f(B)$ is A-measurable for every Borel set $B$ and if $\left(\xi_{j} \subset \mathfrak{B}\right.$ (Borel class on $\Omega$ ) is a sequence of finite partitions of a set $B \in \mathfrak{B}$ such that $\sup \left\{\delta(S) ; S \in \mathbb{B}_{j}\right\}^{107} \rightarrow 0$ as $j \rightarrow \infty$, then the function $n_{B}(P)$, which is defined on o by the number of $p \in B$ such as $f(p)=P$, is $A$ measurable, and for the set function $\lambda(B)$ defined on $\mathfrak{B}$ by $\sup \left\{\sum_{i=1}^{n} A\left(f\left(B_{i}\right)\right) ; \sum_{i=1}^{n} B_{i}=B\right\}$ there holds

$$
\lambda(B)=\lim _{j \rightarrow \infty} \sum_{S \in \mathbb{S} j} \Lambda(f(S))=\int_{0} n_{B}(P) d \Lambda(P) .
$$

From these equalities we know that $\lambda(B)$ is completely additive on $\mathfrak{B}$. The $\lambda$-measurability of sets in $\Omega$ is defined as usual and $\lambda$ becomes a measure on the class of $\lambda$-measurable sets.

We shall say that a not necessarily additive interval function $U(I)$ is absolutely continuous on $I$ if for a given $\varepsilon>0$ there exists $\eta>0$ such that $\sum_{i=1}^{n}\left|U\left(I_{i}\right)\right|<\varepsilon$ whenever the sum of the lengths of non-overlapping intervals $\left\{I_{i}\right\}(i=1, \ldots, n)$ in $I$ is less than $\eta$.

We will give two lemmas concerning the length of a curve.

LEMMA 5.3. If $f(t)$ is a continuous function on $I_{0}: 0 \leqq t \leqq 1$ into a metric space $\sigma$, if $\rho$ is the distance on $\sigma$, if $L$ is the length defined in the usual sense, and if $L\left(I_{0}\right)<+\infty$, then

$$
\lim _{\Delta t \rightarrow 0} \frac{\rho(f(t+\Delta t), f(t))}{\Delta t}
$$

115) M. Brelot [3].

106) H. Federer [1].

10i) $\delta(S)$ denotes the diameter of $S$. 
exists a.e. and, denoting it by $H(t)$, we have $H(t)=L^{\prime}(t)$ a.e. on $I_{0}$ and

$$
\int_{I_{1}} H(t) d t \leqq L\left(I_{1}\right)
$$

for any interval $I_{1} \subset I_{0}$, equality holding if and only if the interval function $U(I)=\rho\left(f\left(t_{1}\right), f\left(t_{2}\right)\right)$, where $I=\left[t_{1}, t_{2}\right]$, is absolutely continuous on $I_{1} \cdot{ }^{108)}$

Proof. The upper Burkill integral of the interval function $U(I)$ gives the usual length $L(I)$ of the curve $\{f(t) ; t \in I\}$, and we can prove the existence of

and the equality

$$
\lim _{\Delta t \rightarrow 0} \frac{\rho(f(t+\Delta t), f(t))}{\Delta t}=H(t) \quad \text { a.e. on } I_{0}
$$

$$
H(t)=L^{\prime}(t) \quad \text { a.e. on } I_{0}
$$

as properties of the Burkill integral. ${ }^{109}$

It is easily shown that the absolute continuities of $L(I)$ and of $U(I)$ are equivalent, and well-known that there holds the inequality

$$
\int_{I_{1}} L^{\prime}(t) d t \leqq L\left(I_{1}\right) \quad \text { for any } I_{1} \subset I_{0},
$$

equality holding if and only if the interval function $L(I)$ is absolutely continuous on $I_{1} \cdot{ }^{110)}$ From these facts the proof will be readily completed.

LEMMA 5.4. If $f(t)$ is a continuous function on $I_{0}: 0 \leqq t \leqq 1$ into a metric space $\sigma$, if $L(I)$ is the length defined in Lemma 5.3, if $A$ is the outer length ${ }^{111}$ ) on $\sigma$, and if $\lambda(B)$ is the measure defined on $B$ in Lemma $5.2,{ }^{112)}$ then there holds

$$
L\left(I_{0}\right)=\lambda\left(I_{0}\right) \text {. }
$$

Proof. There holds for any interval $\left[t_{1}, t_{2}\right]$ in $I_{0}$

$$
\rho\left(f\left(t_{1}\right), f\left(t_{2}\right)\right) \leqq \Lambda\left(\left\{f(t) ; t_{1}<t<t_{2}\right\}\right) \leqq L\left(\left[t_{1}, t_{2}\right]\right){ }^{113)}
$$

Hence if $L\left(I_{0}\right)$ and $\lambda\left(I_{0}\right)$ are finite, then given $\varepsilon>0$ we have for a sufficiently small subdivision of $I_{0}: 0=t_{0}<t_{1}<\ldots<t_{n}=1$,

and

$$
L\left(I_{0}\right)-\varepsilon \leqq \sum_{i=1}^{n} \rho\left(f\left(t_{i-1}\right), f\left(t_{i}\right)\right) \leqq \sum_{i=1}^{n} \Lambda\left(\left\{f(t) ; t_{i-1}<t<t_{i}\right\}\right) \leqq \lambda\left(I_{0}\right)
$$

$$
\lambda\left(I_{0}\right)-\varepsilon \leqq \sum_{i=1}^{n} \Lambda\left(\left\{f(t) ; t_{i-1}<t<t_{i}\right\}\right)^{114)} \leqq \sum_{i=1}^{n} L\left(\left[t_{i-1}, t_{i}\right]\right)=L\left(I_{0}\right),
$$

108) Then we shall say that $f(t)$ is absolutely continuous on $I_{1}$.

109) Cf. S. Saks [1], pp. 165-169, T. Radó [2], Part III, \$1.

110) Cf. S. Saks [1], p. 119.

ii1) C. Carathéodory [2]. Cf. S. Saks [1], p. 54.

112) It is to be remarked here that $f(B)$ is an analytic set and hence 1 -measurable.

113) Cf. S. Saks [1], pp. 123-124.

114) Since $A=0$ for a single point, $A$-measures are equal for open, half open and closed intervals. 
where use is made of Lemma 5.2. Thus $L\left(I_{0}\right)=\lambda\left(I_{0}\right)$. By similar inequalities we can show that the infinity of one induces the infinity of the other.

\section{Rectifiability of boundary of a Riemann surface}

In this chapter let $\mathfrak{R}$ be a simply-connected Riemann surface of hyperbolic type as an inner covering surface over a metric Riemann surface $\sigma$. Let the distance on $\sigma$ be $\rho\left(P_{1}, P_{2}\right)$ and assume that at every point $P \in \sigma$ there exists a neighborhood $N$ and a local parameter $w$ such that

$$
0<m<\frac{\rho\left(P_{1}, P_{2}\right)}{\left|w\left(P_{1}\right)-w\left(P_{2}\right)\right|} \equiv \kappa\left(w\left(P_{1}\right), w\left(P_{2}\right)\right)<M<+\infty \text { 113) }
$$

for $P_{1} \neq P_{2} \in N$, where $m$ and $M$ may depend upon $P$ and $w$. If $\omega$ is another local parameter of $N$, a similar inequality holds in such neighborhood $N^{\prime}$ of $P$ as $N^{\prime a} \subset N$ also for $\kappa\left(\omega\left(P_{1}\right), \omega\left(P_{2}\right)\right)$, which is defined similarly as in (5.1), because $w^{\prime}(\omega) \neq 0$ on $N^{\prime}$ and hence

$$
0<m^{\prime}<\frac{\left|w\left(P_{1}\right)-w\left(P_{2}\right)\right|}{\left|\omega\left(P_{1}\right)-\omega\left(P_{2}\right)\right|}<M^{\prime}<+\infty
$$

for $P_{1} \neq P_{2} \in N^{\prime}$.

Let us consider the accessible boundary points of $\Re$. A curve on $\Re$, which terminates at two different accessible boundary points, is called a cross-cut and divides $\Re$ into two parts. Take an infinite sequence of cross-cuts $\left\{q_{n}\right\}$ on $\Re$, whose end-points are different from each other and whose projections on $\sigma$ tend to a point as $n \rightarrow \infty$. Further suppose that $q_{n-1}$ and $q_{n+1}$ lie in different parts, into which $\Re$ is divided by $q_{n}$, and denote the part which contains $q_{n+1}$ by $\Re_{n}$. We designate such a sequence $\left\{\Re_{n}\right\}$ a fundamental one. Two fundamental sequences $\left\{\Re_{n}\right\}$ and $\left\{\mathfrak{R}_{n}^{\prime}\right\}$ are called equivalent when for any assigned integer $m$ there exists an integer $n$ such that $\Re_{m} \supset \Re_{n}^{\prime}$ and $\Re_{m}^{\prime} \supset \Re_{n}$. To every class of equivalent fundamental sequences let a boundary element be made to corre: spond.116) Now we can introduce a topology into the space consisting of $\Re$ and the set $\mathfrak{F}_{\Re}$ of all the boundary elements quite similarly as in Chap. III, $\S 5$. The intersection of the closures of the projections on $\sigma$ of $\left\{\mathfrak{R}_{n}\right\}$, which determine a boundary element $P_{\mathfrak{F}}$, will be called the projection of $P_{\mathbb{F}}$, and $P_{\mathbb{F}}$ will be said to lie over $P$ when $P$ belongs to the projection of $P_{(5)}$.

If $\Re+\xi_{\Re}$ is a compact space, ${ }^{117}$ and if there holds

115) This assumption expresses that the distance $\rho$ is comparable locally with the euclidean distance.

116) C. Carathéodory defined this for a plane domain in [1].

117) $\Re+E_{\mathfrak{F}}$ is not necessarily compact. Compare this with ${ }^{72)}$. It can be shown that it is compact when the maximal covering number of $\Re$ over $\sigma$ is finite. 


$$
\int_{0} n(P) d \Lambda(P)<+\infty,{ }^{118)}
$$

where $n(P)$ is the number of the boundary elements lying over $P$ and $\Lambda$ is the outer length on $\sigma$, then we shall say that the boundary of $\mathfrak{A}$ is rectifiable.

\section{Continuity of the mapping function}

Hereafter we suppose that the boundary of $\Re$ is rectifiable. Mapping $U$ : $|z|<1$ onto $\Re$ conformally and taking the projection $\Re \rightarrow \sigma$, we denote the mapping function of $U$ into $\sigma$ by $f(z)$. For this function we have

THEOREM 5.1. $f(z)$ can be defined also on $|z| \leqq 1$ so that it is still continuous there.

Proof. The part of $\sigma$ over which the boundary elements lie is equal to $\bigcap_{n}\left(f\left(D_{n}\right)\right)^{a, 119)}$ where $D_{n}$ is the ring domain $1-\frac{1}{n}<|z|<1$. As that part is closed and has finite $\Lambda$-measure by $(5.2), f(z)$ does not take values of an open set on $\sigma$ in $U$ sufficiently near $\Gamma$. Then it is easy to show the one-to-one correspondence between $\mathfrak{F}_{\Re}$ and $\Gamma$ by making use of Lindelöf's and Koebe's theorems. ${ }^{120)}$ Thus $n(P)$ is equal to such a number of $z \in \Gamma$ as $P \in S_{z}^{(U)}$.

Let $F$ be a closed arc on $\Gamma$, and take a sequence of cross-cuts in $U$ which cut off open arcs $\left\{G_{n}\right\}$ from $\Gamma$ and $\left\{U_{n}\right\}$ from $U$ such that $G_{n} \downarrow F$ and $U_{n}^{a} \downarrow F$. Then $f\left(U_{n}\right)^{a} \downarrow S_{F}$ as $n \rightarrow \infty$, where $S_{F}=\bigcup_{z \in F} S_{z}^{\left({ }^{*}\right)}$. Therefore $S_{F}$ is a continuum or a point on $\sigma$. When $E$ is a half open arc, $S_{E}$ is the union of an enumerably infinite number of closed sets and hence $\Lambda$-measurable.

Let $z_{0}$ be any point on $\Gamma$, take a sequence of open arcs $\left\{G_{n}\right\}$ such that $G_{n} \downarrow z_{0}$, put $G_{n}-G_{n+1}=E_{n}^{(1)}+E_{n}^{(2)}$, where $E_{n}^{(1)}$ and $E_{n}^{(2)}$ are half open arcs lying on each side of $z_{0}$, and put $\sum_{k=n}^{\infty} E_{k}^{(i)}=\Delta_{n}^{(i)}(i=1,2)$. Since by (5.2)

$$
\sum_{k=1}^{\infty}\left(\Lambda\left(S_{E_{k}^{(1)}}\right)+\Lambda\left(S_{E_{k}^{(2)}}\right)\right)<+\infty,
$$

$\Lambda\left(S_{S_{n}^{(1)}}\right) \leqq \sum_{k=n}^{\infty} \Lambda\left(S_{E_{k}^{(1)}}\right)$ tends to zero as $n \rightarrow \infty$. Then we can assert that the diameter of $S_{\Delta_{n}^{(1)}}$ tends to zero as $n \rightarrow \infty$. For if the diameter did not tend to zero, there would be two points of $S_{\lrcorner_{n}^{(1)}}$ for all $n$, which have positive distance $\rho_{0}$ to each other. Then $A$-measure of $S_{\perp_{n}^{(1)}}$ would be not less than $\rho_{0}>0$ for all $n$ and this is a contradiction. Now $S_{z_{0}}^{\left(r_{1}\right)_{121)}}=\bigcap_{n}\left(S_{\Delta_{n}^{(1)}}^{(a}\right.$ turned out to be a single

119) For the definition of lower integral see Chap. IV, $\S 1$.

19) $\left(f\left(D_{n}\right)\right)^{a}$ means the closure of the set $\left\{f(z) ; z \in D_{n}\right\}$.

120) Is it possible to show the one-to-one correspondence without using the condition (5.2)?

121) $\Gamma_{1}$ and $\Gamma_{2}$ are two sides of $\boldsymbol{z}_{0}$ on $\Gamma$. 
point. The same is true of $S_{z_{0}}^{\left(r_{2}\right)}$. As it is seen previously that $S_{z_{0}}^{(U)}$ is not identical with $\sigma$, there holds

$$
S_{z_{0}}^{\left(\Gamma_{1}\right)}=S_{z_{0}}^{\left(\Gamma_{2}\right)}=S_{z_{0}}^{(U)}=\text { one point }
$$

by Lemma 5.1. By the arbitrariness of $z_{0}, f(z)$ can be defined so as to be continuous everywhere on $|z| \leqq 1$.

\section{Correspondence of null sets}

We shall denote the set $\left\{z \in T ; f(z) \in E^{\prime}\right\}$ for a set $E^{\prime}$ on $\sigma$ by $f^{-1}\left(E^{\prime}\right)$ and say that $f^{-1}\left(E^{\prime}\right)$ on $\Gamma$ corresponds to $E^{\prime}$.

When a function is bounded regular in $U$ and continuous on $U+\Gamma$ and the image of $I$ is rectifiable, it was shown by $F$. and $M$. Riesz ${ }^{122}$ ) that the image of a null set on $\Gamma$ has a linear measure zero and vice versa. Also in the present case we can prove

THEOREM 5.2. Under the same condition as in $\S 4$ the null sets on $I$ and the null sets on o correspond to each other.

Proof. First we assume that $f(z)$ is absolutely continuous on $\Gamma$. Since $\lambda\left(I_{0}\right)=\int_{0} n(P) d \Lambda(P)<+\infty\left(I_{0}=[0,2 \pi]\right)$ by Lemma 5.2 and $(5.2)$, it follows by Lemmas 5.3 and 5.4 that there exists $H(\theta)=\lim _{\Delta \theta \rightarrow 0} \rho\left(f\left(e^{i(\theta+\Delta \theta)}\right), f\left(e^{i \theta}\right)\right) / \Delta \theta$ a.e. on $r$ and holds

$$
\lambda(I)=L(I)=\int_{I} H(\theta) d \theta,
$$

where $I=\left[\theta_{1}, \theta_{2}\right]$ and $L(I)$ is the length of the image on $\sigma$ of $e^{i \theta_{1}} e^{i \theta_{2}}$. From (5.3) follows $\lambda(G)=\int_{G} H(\theta) d \theta$ for any open set $G \subset \Gamma$. Further holds for any Borel set $B$

$$
\lambda(B)=\inf \{\lambda(G) ; B \subset G\}^{123)}=\inf \left\{\int_{G} ; B \subset G\right\}=\int_{B} .
$$

Taking account of Lemma $5.2 n_{B}(P)$ is $\Lambda$-measurable and

$$
\int_{\sigma} n_{B}(P) d A(P)=\int_{B} H(\theta) d \theta .
$$

Now let $E$ be a null set on $\Gamma$. Then there exists a $G_{\delta}$ null set $E_{1} \supset E$ and (5.4) holds for $E_{1}$. Hence $\int_{\sigma} n_{E_{1}} d \Lambda=0$. Since $f\left(E_{1}\right)$ is equal to $\left\{P ; n_{E_{1}}(P) \equiv 1\right\}$, $A\left(f\left(E_{1}\right)\right)=0$ whence also $A(f(E))=0$. Thus a null set on $\sigma$ corresponds to a null set on $I$.

Next suppose that $H(\theta)$ vanishes only on a null set on $\Gamma$. If $E^{\prime}$ is a null

129. See ${ }^{26)}$.

123) Cf. A. P. Morse and J. P. Randolph [1]. 
set on $\sigma$, there exists a $G_{\delta}$ null set $E_{1}^{\prime} \supset E^{\prime 29 ?)}$ and $f^{-1}\left(E_{1}^{\prime}\right)$ is a $G_{\delta}$ set on $\Gamma$. Therefore (5.4) holds for $f^{-1}\left(E_{1}^{\prime}\right)$. Since $\int_{0} n_{f^{-1}\left(E_{1}^{\prime}\right)} d A=\int_{E_{1}^{\prime}} n d \Lambda=0$, there follows $\int_{f^{-1}\left(E_{1}^{\prime}\right)} H(\theta) d \theta=0$. Hence $m\left(f^{-1}\left(E_{1}^{\prime}\right)\right)=m\left(f^{-1}\left(E^{\prime}\right)\right)=0$. Thus a null set on $\Gamma$ corresponds to a null set on $\sigma$.

In the following we shall show that $f(z)$ is absolutely continuous on $\Gamma$ and $H(\theta)$ vanishes only on a null set on $\Gamma$.

As we have seen in the proof of Theorem 5.1, near $\Gamma f(z)$ does not take values of an open set on $\sigma$. Hence we can map the universal covering surface of $\sigma$ on the $w$-plane such that, taking a branch, the meromorphic function $w(z)$, which corresponds to the mappings $U \rightarrow \sigma \rightarrow w$-plane, is bounded near $T$. It is resolved in $U$ in the form:

$$
w(z)=\left(\prod_{k=1}^{n} \frac{1-\bar{\alpha}_{k} z}{z-\alpha_{k}}\right) g(z)=h(z) g(z),
$$

where $\left\{\alpha_{k}\right\}$ are poles of $w(z)$ in $U$ and $g(z)$ is regular in $U$ and continuous on $\Gamma$.

We shall evaluate the usual length $L_{g}\left(I_{0}\right)$ of the curve $\left\{g\left(e^{i \theta}\right) ; \theta \in I_{0}\right\}$ on the $w$-plane. $w$ may be taken as a local parameter for every $N$ in $\S 3$. Since the $w$-image of $\Gamma$ is closed in the $w$-plane, it is covered by a finite number of the $w$-images of such $N^{\prime}$ as $N^{\prime a} \subset N$. Let the selected $N^{\prime}$ be $\left\{N_{i}\right\} \quad(i=1,2, \ldots$, $n$ ). Since (j.1) holds in every $N_{i}$ for the selected branches, there exist $m$ and $M$ such that

$$
0<m<\kappa\left(w\left(P_{1}\right), w\left(P_{\mathrm{a}}\right)\right)<M<+\infty,
$$

where $P_{1}$ and $P_{:}\left(P_{1} \neq P_{2}\right)$ belong to some one $N_{i}$ and $w\left(P_{1}\right)$ and $w\left(P_{2}\right)$ are the images by a certain branch selected for $N_{i}$. Since $f\left(e^{i \theta}\right)$ is continuous on $I$, we can take a subdivision of $\Gamma$ such that the image of every piece lies in a cartain $N_{i}$. For such a subdivision we have by (5.5)

$$
\begin{aligned}
\sum_{k=1}^{p}\left|g\left(e^{i \theta_{k-1}}\right)-g\left(e^{i \theta_{k}}\right)\right| & =\sum_{k=1}^{p} \frac{w\left(e^{i \theta_{k}-1}\right)}{h\left(e^{i \theta_{k-1}}\right)}-\frac{w\left(e^{i \theta_{k}}\right)}{h\left(e^{i \theta_{k}}\right)}=\sum_{k=1}^{p} \mid w\left(e^{i \theta_{k-1}}\right) h\left(e^{i \theta_{k}}\right) \\
-w\left(e^{i \theta_{k}}\right) h\left(e^{i \theta_{k-1}}\right) \mid & =\sum_{k=1}^{p}\left|w\left(e^{i \theta_{k-1}}\right)-w\left(e^{i \theta_{k}}\right)\right|+\sum_{k=1}^{p}\left|w\left(e^{i \theta_{k}}\right)\right|\left|h\left(e^{i \theta_{k-1}}\right)-h\left(e^{i \theta_{k}}\right)\right| .
\end{aligned}
$$

From (5.6)

the first sum $=\sum_{k=1}^{v} \frac{\rho\left(f\left(e^{i \theta_{k-1}}\right), f\left(e^{i \theta_{k}}\right)\right)}{\kappa\left(w\left(e^{i \theta_{k-1}}\right), w\left(e^{i \theta_{k}}\right)\right)} \leqq \frac{1}{m} \sum_{k=1}^{r} \rho\left(f\left(e^{i \theta_{k-1}}\right), f\left(e^{i \theta_{k}}\right)\right) \leqq \frac{1}{m} L\left(I_{0}\right)<+\infty$ (such terms as $w\left(e^{i \theta_{k-1}}\right)=w\left(e^{i \theta_{k}}\right)$ are excluded),

the second sum $\leqq M_{1} \sum_{k=1}^{p}\left|h\left(e^{i \theta_{k-1}}\right)-h\left(e^{i \theta_{k}}\right)\right| \leqq M_{1} L_{h}\left(I_{0}\right)$, 
where $L_{h}\left(I_{0}\right)$ is the length of the curve $\left\{h\left(e^{i \theta}\right) ; \theta \in I_{0}\right\}$, and is finite, since $h(z)$ is regular also on $T$. Therefore $L_{g}\left(I_{0}\right)=\sup \sum_{k=1}^{p}\left|g\left(e^{i \theta_{k-1}}\right)-g\left(e^{i \theta_{k}}\right)\right|$ is finite.

This once established, it is possible by the usual method ${ }^{124)}$ to show that $g^{\prime}(z)$ is of bounded type in $U$, that $g\left(e^{i \theta}\right)$ is absolutely continuous and that $g^{\prime}\left(e^{i \theta}\right)$ exists a.e. on $\vec{i}$. Therefore $w^{\prime}(z)=g^{\prime}(z) h(z)+g(z) h^{\prime}(z)$ is of bounded type and $w^{\prime}\left(e^{i \theta}\right)$ exists a.e. on $I$. Furthermore since for such $\theta$ and $\theta^{\prime}$ $\left(0 \leqq \theta<\theta^{\prime}<2 \pi\right)$ as $f\left(\widehat{e^{i \theta} e^{i \theta^{\prime}}}\right)$ lies in a certain $N_{i}$ and $f\left(e^{i \theta}\right) \neq f\left(e^{i \theta^{\prime}}\right)$ there holds

$$
\begin{aligned}
& \rho\left(f\left(e^{i \theta}\right), f\left(e^{i \theta^{\prime}}\right)\right)=\kappa\left(w\left(e^{i \theta}\right), w\left(e^{i \theta^{\prime}}\right)\right)\left|w\left(e^{i \theta}\right)-w\left(e^{i \theta^{\prime}}\right)\right| \\
& \quad \leqq M\left|w\left(e^{i \theta}\right)-w\left(e^{i \theta^{\prime}}\right)\right| \leqq M\left|g\left(e^{i \theta}\right)-g\left(e^{i \theta^{\prime}}\right)\right|+M M_{1}\left|h\left(e^{i \theta}\right)-h\left(e^{i \theta^{\prime}}\right)\right|
\end{aligned}
$$

and $g\left(e^{i \theta}\right)$ and $h\left(e^{i \theta}\right)$ are absolutely continuous, $f\left(e^{i \theta}\right)$ is also absolutely continuous on $\Gamma$.

Since on account of Lemma $5.3 H(\theta)$ exists a.e. on $\Gamma$, also

$$
\lim _{\Delta \theta \rightarrow 0} \kappa\left(w\left(e^{i(\theta+\Delta \theta)}\right), w\left(e^{i \theta}\right)\right)=H(\theta) /\left|w^{\prime}\left(e^{i \theta}\right)\right|
$$

exists a.e. on $\Gamma$. If we denote this by $\kappa_{w}(\theta)$, there holds

$$
H(\theta)=\kappa_{w}(\theta)\left|w^{\prime}\left(e^{i \theta}\right)\right| .
$$

Since $w^{\prime}(z)$ is of bounded type ${ }^{155)}$ and $\kappa_{w}(\theta) \geqslant m>0$ by $(5.6), H(\theta)$ vanishes only on a null set. Thus the proof is completed.

This theorem may be regarded as a generalization of F. and M. Riesz's theorem and be stated in the following form in connexion with Chap. IV:

THEOREM 5.3. On a Riemann surface with a rectifiable boundary Dirichlet problem is manageable by the procedure in Chap. IV, §2, and a set on the boundary has harmonic measure zero if and only if its projection on o has zero A-measure.

This is applicable, for instance, to the following theorem due to P. J. Myrberg: ${ }^{126}$ )

Let $\left\{a_{n}\right\}$ be a sequence of points in $|w|<1$ and radial slits be drawn from every $a_{n}$ to $r:|w|=1$. If the total length of the slits is finite, the harmonic measure of $r$ with respect to the slit domain is positive.

It is known that if the total length is infinite there appear cases in which the assertion of this theorem is not true. ${ }^{127)}$ Hence the condition (5.2) is necessarily to be imposed.

Let $D$ be a plane domain outside a set $C$ of positive capacity, but of outer 124) Cf. F. Riesz [1], M. Tsuji [1].

125) See the extension of F. and M. Riesz's theorem in p. 197 of R. Nevanlinna [2].

126) P. J. Myrberg [2].

127) Ibid. and N. Lusin and J. Priwaloff [1]. 
length (linear measure) zero. When the universal covering surface of $D$ is mapped conformally onto $U, C$ corresponds to a set of linear measure $2 \pi$ on $\Gamma$. From this example we know that the compactness of $\Re+\mathfrak{E}_{\mathfrak{R}}$ is necessary for the validity of Theorem 5.3, even if Dirichlet problem is manageable on the Riemann surface by the procedure in Chap. IV, $\$ 2$.

\section{Equalities for the length}

We obtained some equalities for the lengths of sets on $I$ in $\S 5$. Now we shall complete them in this section.

Let $E$ be any linearly measurable set on $I$. Then there exist an $F_{0}$ set $E_{1}$ and a $G_{\delta}$ set $E_{2}$ such that $E_{1} \subset E \subset E_{2}$ and $m E_{1}=m E=m E_{2}$. From (5.4) we know that $\int_{0} n_{E_{1}} d A=\int_{0} n_{E_{2}} d \Lambda$ and this shows the $A$-measurability of the function $n_{E}(P)$, because $n_{E_{1}}(P) \leqq n_{E}(P) \leqq n_{E_{2}}(P)$ and both $n_{E_{1}}(P)$ and $n_{E_{2}}(P)$ are A-measurable. Further there holds

$$
\int_{\sigma} n_{E}(P) d \Lambda(P)=\int_{E} H(\theta) d \theta
$$

It can be proved similarly that for any 1 -measurable set $E^{\prime} f^{-1}\left(E^{\prime}\right)$ is linearly measurable on $\Gamma$.

From (5.7) and (5.8) there follows

$$
\int_{\sigma} n_{E}(P) d A(P)=\int_{E} \kappa_{w}(\theta)\left|w^{\prime}\left(e^{i \theta}\right)\right| d \theta
$$

for any linearly measurable set $E \subset \Gamma$. We can show this equality also in the case when $\sigma$ is mapped on the $w$-plane so that the function $w(z)$ on $U$ is not bounded near $\Gamma$, i.e., $w(z)=\infty$ at some points on $\Gamma$.

Finally let $W=\Psi(P)$ be a non-constant one-valued meromorphic function on $\sigma$ and denote the function $\Psi(f(z))$ by $W(z)$. Then also in this case $W^{\prime}\left(e^{i \theta}\right)$ and $\kappa_{W}(\theta)=\lim _{\Delta \theta \rightarrow 0} \kappa\left(W\left(e^{i(\theta+\Delta 0)}\right), W\left(e^{i \theta}\right)\right)$ exist a.e. on $I^{\prime}$ and there holds for any linearly measurable set $E$

$$
\int_{\sigma} n_{E}(P) d A(P)=\int_{E} \kappa_{W}(\theta)\left|W^{\prime}\left(e^{i \theta}\right)\right| d \theta .
$$

Examples. The variables $w$ and $W$ in examples are supposed to have the same meaning as above.

1) Let $\sigma$ be a simply-connected Riemann surface of elliptic or parabolic type and define the distance $\rho\left(P_{1}, P_{s}\right)$ by $\left|w\left(P_{1}\right)-w\left(P_{2}\right)\right| / \sqrt{\left(1+\left|w\left(P_{1}\right)\right|^{2}\right)\left(1+\mid w\left(P_{s}\right)_{1}^{! 2}\right)}$. Then $\kappa_{w}(\theta)=1 /\left(1+\left|w\left(e^{i 0}\right)\right|^{2}\right)$.

2) Let $\sigma$ be a closed surface of the genus 1 and define the distance $\rho\left(P_{1}\right.$, $\left.P_{2}\right)$ by $\min \left|w\left(P_{1}\right)-w\left(P_{2}\right)\right|$, where $w\left(P_{1}\right)$ and $w\left(P_{2}\right)$ represent the points corresponding to $P_{1}$ and $P_{2}$ respectively on the $w$-plane. Then $\kappa_{w}(\theta)=1$. 
3) Let $\sigma$ be a Riemann surface different from those stated in 1) and 2), and define the distance $\rho\left(P_{1}, P_{2}\right)$ by $\min \left|w\left(P_{1}\right)-w\left(P_{2}\right)\right| /\left|1-w\left(P_{1}\right) \overline{w\left(P_{2}\right)}\right|$, where $w\left(P_{1}\right)$ and $w\left(P_{2}\right)$ represent the points corresponding to $P_{1}$ and $P_{2}$. Then $\kappa_{w}(\theta)=1 /(1$ $\left.-\left|w\left(e^{i \theta}\right)\right|^{2}\right)$. If the distance is given by $\log \left(\left(1+\rho\left(P_{1}, P_{2}\right)\right) /\left(1-\rho\left(P_{1}, P_{2}\right)\right)\right)$, the value of $\kappa$ doubles.

4) Let $\sigma$ be a covering surface over the Riemann sphere and define the distance $\rho\left(P_{1}, P_{2}\right)$ as in Teichmüller's metric. ${ }^{129)}$ Then locally $\rho\left(P_{1}, P_{2}\right)=\mid W\left(P_{1}\right)$ $-W\left(P_{2}\right) \mid / \sqrt{\left(1+\left|W\left(P_{1}\right)\right|^{2}\right)\left(1+\left|W\left(P_{2}\right)\right|^{2}\right)}$ except over $W=\infty$ or at the branch points of $\sigma$ and $\kappa_{W}(\theta)=1 /\left(1+\left|W\left(e^{i \theta}\right)\right|^{2}\right)$.

5) Let $\sigma$ be a Riemann surface regarded as a Finsler space, $d s^{2}=F(x, y$, $d x, d y)$ the infinitesimal distance represented in a local parameter circle, and $C=\{P(t) ; 0 \leqq t \leqq 1\}$ a continuous curve on $\sigma$, whose image in any parameter circle is absolutely continuous and rectifiable in the usual sense. Then the integral arc length $\int_{C}$ of $C$ will be defined by $\int_{0}^{1} \sqrt{F\left(x, y, \frac{d x}{d t}, \frac{d y}{d t}\right)} d t$ as a Lebesgue integral, and a metric on $\sigma$ will be introduced by distance $\rho\left(P_{1}, P_{2}\right)$ $=\inf \int_{C}$, where $C$ is a curve possessing the properties stated above and joins $P_{1}$ with $P_{2}$. We shall call this the geodesic distance. It is known that this metric gives the topology equivalent to the original one of $\sigma$, that it satisfies the inequality (5.1), and that the $L$-length defined by this metric is equal to $\int_{c}$ for any $C$ mentioned above. ${ }^{129)} \quad$ Further there holds $d \int_{0}^{t} / d t=\sqrt{F\left(x, y, \frac{d x}{d t}, \frac{d y}{d t}\right)}$ a.e. on $[0,1]$.

Applying these results we have from $(5.3)$ and (5.7) a.e. on $\Gamma$

$$
\sqrt{F\left(x, y, \frac{d x}{d \theta}, \frac{d y}{d \theta}\right)}=\kappa_{w}(\theta)\left|w^{\prime}\left(e^{i 0}\right)\right|,
$$

where $w=x+i y$. The same is true of the variable $W$. In examples 1) to 4 ) every $\sigma$ can be regarded as a Riemann space with the infinitesimal distance of the form: $d s=\lambda(w)|d w|$ or $\lambda(W)|d W|$. Then $\kappa_{w}(\theta)=\lambda\left(w\left(e^{i \theta}\right)\right)$ or $\kappa_{W}(\theta)$ $=\lambda\left(W\left(e^{i \theta}\right)\right)$ a.e. on $\Gamma$. The geodesic distance is equal to an arc length along a great circle on a Riemann sphere in 1), to $\rho\left(P_{1}, P_{2}\right)$ in 2) and 4), and to $\log \left(\left(1+\rho\left(P_{1}, P_{s}\right)\right) /\left(1-\rho\left(P_{1}, P_{2}\right)\right)\right.$ in 3$)$.

128) Cf. Chap. III, § 1.

129) Cf. M. Morse [1], pp. 67-68, S. B. Myers [1]. 


\section{BIBLIOGRAPHY}

L. Ahlfors:

(* is referred to only through Math. Rev.)

[1] Über die Anwendung differentialgeometrischer Methoden zur Untersuchung von Überlagerungsflächen, Acta Soc. Sci. Fenn., N. s. A. 2 (1937), No.6, 17 pp.

[2] Zur Uniformisierungstheorie, 9 Congrès Math. Scand. Helsingfors, (1939), pp. 235248.

[3] Die Begründung des Dirichletschen Prinzips, Soc. Sci. Fenn. Comment. Phys.-math., 11 (1943), No. 15, $15 \mathrm{pp}$.

[4]* Das Dirichletsche Prinzip, Math. Ann., 120 (1947), pp. 36-42.

R. Bader:

[1] La théorie du potentiel sur une surface de Riemann, C. R. Acad. Sci. Paris, 228 (1949), pp. 2001-2002.

L. Bieberbach:

[1] Lehrbuch der Funktionentheorie, II, Leipzig and Berlin, 1 Aufl. (1927), 2 Aufl. (1931). M. Brelot:

[1] Problème de Dirichlet et majorantes harmoniques, Bull. Sci. Math., (2), 63 (1939), pp. 79-96 and 115-128.

[2] Familles de Perron et problème de Dirichlet, Acta Univ. Szeged, 9 (1939), pp.133153.

[3] Sur l'allure à la frontière des fonctions harmoniques, sousharmoniques ou holomorphes, Bull. Soc. Roy. Liège, (1939), pp. 468-477.

[4] Sur la théorie autonome des fonctions sousharmoniques, Bull. Sci. Math., (2), 65 (1941), pp. 72-98.

[5] Le problème de Dirichlet "ramifié," Ann. Grenoble, 22 (1946), pp. 167-200.

C. Carathéodory:

[1] Über die Begrenzung einfach zusammenhängender Gebiete, Math. Ann., 73 (1913), pp. 323-370.

[2] Über das lineare Mass von Punktmengen-eine Verallgemeinerung des Längenbegriffs, Nachr. Gött., (1914), pp. 404-426.

[3] On Dirichlet's problem, Amer. J. Math., 59 (1937), pp. 709-731.

G. C. Evans:

[1] Potentials and positively infinite singularities of harmonic functions, Monat. Math. Phys., 43 (1936), pp. 419-424.

H. Federer:

[1] Surface area, I, Trans. Amer. Math. Soc., 55 (1944), pp. 420-437.

O. Frostman:

[1] Potentiel d'équilibre et capacité des ensembles avec quelques applications à la théorie des fonctions, Meddel. Lunds. Univ. Mat. Sem., 3 (1935), pp. 1-118.

M. H. Heins:

[1] The conformal mapping of simply-connected Reimann surfaces, Ann. of Math., 50 (1949), pp. 686-690. 
F. Iversen:

[1] Sur quelques propriétés des fonctions monogènes au voisinage d'un point singulier, Öfv. af Finska Vet.-Soc. Förh., A. 58 (1916), No. 25, 17 pp.

G. Julia:

[1] Leçons sur la représentation conforme des aires multiplement connexes, Paris (1934).

E. Kaila:

[1] Über die Ränderzuordnung bei konformer Abbilding von mehrfachzusammenhängenden Gebieten, Ann. Acad. Sci. Fenn., (A) 55 (1940), No. 9, 63 pp.

S. Kametani and T. Ugaheri:

[1] A remark on Kawakami's extension of Löwner's lemma, Proc. Imp. Acad. Tokyo, 18 (1942), pp. 14-15.

Y. Kawakami:

[1] On an extension of Löwner's lemma, Jap. J. Math., 17 (1941), pp. 569-572.

B. v. Kerékjártú:

[1] Vorlesungen über Topologie, Berlin (1923).

K. Kodaira:

[1] Theory of Riemann surfaces, Kôsû Kenkyu, 7-8 (1943), (in Japanese).

[2] Harmonic fields in Riemannian manifolds, Ann. of Math., 50 (1949), pp. 587-665.

P. Koebe:

[1] Über die Uniformisierung beliebiger analytischer Kurven, III, Nachr. Gött., (1908), pp. 337-358.

K. Kunugui:

[1] Sur un théorème de M. M. Seidel-Beurling, Proc. Imp. Acad. Tokyo, 15 (1939), pp. $27-32$.

K. Lőwner:

[1] Untersuchungen über schlichte konforme Abbildungen des Einheitskreises, Math. Z., 3 (1919), pp. 103-121.

N. Lusin and J. Priwalof:

[1] Sur l'unicité et la multiplicité des fonctions analytiques, Ann. École Norm., 42 (1925), pp. 143-191.

A. P. Morse and J. P. Randolph:

[1] The $\phi$-rectifiable subsets of the plane, Trans. Amer. Math. Soc., 55 (1944), pp. 236305.

M. Morse:

[1] Functional topology and abstract variational theory, Paris (1938).

S. E. Myers:

[1] Arc length in metric and Finsler manifolds, Ann. of Math., 39 (1938), pp. 463-472.

P: J. Myrberg:

[1] Über die Existenz der Greenschen Funktionen auf einer gegebenen Riemannschen Fläche, Acta Math., 61 (1933), pp. 39-79.

[2] Über den Fundamentalbereich der automorphen Funktionen, Ann. Acad. Sci. Fenn., A. I, (1941), No. 2, $25 \mathrm{pp}$. 
C. Neumann:

[1] Vorlesungen über Riemanns Theorie der Abelschen Integrale, 2 Aufl. Leipzig (1884). R. Nevanlinna:

[1] Das harmonische Mass von Punktmengen und seine Anwendung in der Funktionentheorie, 8 Congrès Scand. Stockholm, (1934), pp. 116-133.

[2] Eindeutige analytische Funktionen, Berlin (1936).

[3] Über die Lösbarkeit des Dirichletschen Problems für eine Riemannsche Fläche, Nachr. Gött., 1 (1938), pp. 181-193.

[4] Ein Satz über offene Riemannsche Flächen, Ann. Acad. Sci. Fenn., (A) 54 (1940), No. 3, 16 pp.

[5] Quadratisch integrierbare Differentiale auf einer Riemannschen Mannigfaltigkeit, Ann. Acad. Sci. Fenn., A. I, (1941), No. 1, 34 pp.

[6] Über das Anwachsen des Dirichletintegrals einer analytischen Funktion auf einer offenen Riemannschen Fläche, ibid., (1948), No. 45, 9 pp.

[7]* Über die Neumannsche Methode zur Konstruktion von Abelschen Integrale, Comment. Math. Helv., 22 (1949), pp. 302-316.

[8] Über die Anwendung einer Klasse von Integralgleichungen für Existenzbeweise in der Potentialtheorie, Acta Univ. Szeged, 12, A, (1950), pp. 146-160.

M. Ohtsuka:

[1] A theorem on the Poisson integral, Proc. Japan Acad., 22 (1946), pp. 195-197.

W. F. Osgood:

[1] Lehrbuch der Funktionentheorie, II, 2, Leipzig and Berlin (1932).

M. Parreau:

[1] Comportement à la frontière de la fonction de Green d'une surface de Riemann, C. R. Acad. Sic. Paris, 230 (1950), pp. 709-711.

[2] La théorie du potentiel sur les surfaces de Riemann à frontière positive, ibid., pp. 914-916.

[3] Sur les moyennes des fonctions harmoniques et-la classification des surfaces de Riemann, ibid., 231 (1950), pp. 679-681.

O. Perron:

[1] Eine neue Behandlung der ersten Randwertaufgabe für $\Delta \boldsymbol{u}=0$, Math. Z., 18 (1923), pp. 42-54.

T. Radó:

[1] Über den Begriff der Riemannschen Fläche, Acta Univ. Szeged, 2 (1925), pp. 101-121.

[2] Length and area, New York (1948).

T. Radó and F. Riesz:

[1] Über erste Randwertaufgabe für $\boldsymbol{s} \boldsymbol{u}=0$, Math. Z., 22 (1925), pp. 41-44.

R. Remak:

[1] Über potentialkonvexe Funktionen, Math. Z., 20 (1924), pp. 126-130.

F. Riesz:

[1] Über die Randwerte einer analytischen Funktion, Math. Z., 18 (1923), pp. 87-95. 
F. and M. Riesz:

[1] Über die Randwerte einer analytischen Funktion, 4 Congrès Scand. Stockholm, (1916), pp. $27-44$,

S. Saks:

[1] Theory of the integral, Warsaw (1937).

L. Sario:

[1] Existence des fonctions d'allure donnée sur une surface de Riemann arbitraire, C. $R$. Acad. Sci. Paris, 229 (1949), pp. 1293-1295.

[2] Quelques propriétés à la frontière se rattachant à la classification des surfaces de Riemann, ibid., 230 (1950), pp. 42-44.

W. Seidel:

[1] Über die Ränderzuordnung bei konformer Abbildung, Math. Ann., 104 (1931), pp. $182-243$

[2] On the distribution of values of bounded analytic functions, Trans. Amer. Math. Soc., 36 (1934), pp, 201-226.

S. Stoilow:

[1] Leçons sur les principes topologiques de la théorie des fonctions analytiques, Paris (1938).

J. Tamura:

[1] On the existence of automorphic functions, Math. Report of Todai-Kyoyogakubu, $\mathbf{1}$ (1950), pp.157-164, (in Japanese).

O. Teichmüller:

[1] Erreichbare Randpunkte, Deut. Math., 4 (1939), pp. 455-461.

M. Tsuji:

[1] A theorem on conformal mapping, Jap. J. Math., 17 (1940), pp. 97-108.

[2] On an extension of Löwner's theorem, Proc. Imp. Acad. Tokyo, 18 (1942), pp. 220-221.

[3] Theory of meromorphic function in a neighbourhood of a closed set of capacity zero, Jap. J. Math., 19 (1944), pp. 139-154.

[4] Some metrical theorems on Fuchsian groups, Jap. J. Math., 19 (1947), pp. 483-516.

K. I. Virtanen:

[1] C̈ber die Existenz von beschränkten harmonischen Funktionen auf offenen Riemannschen Flächen; Ann. Acad. Sci. Fenn., A. I., (1950), No.75, 7 pp.

H. Weyl:

[1] Die Idee der Riemannschen Fläche, Leipzig and Berlin (1913).

[2] The method of orthogonal projection in potential theory, Duke Math. J., 7 (19.40), pp. $411-444$.

\section{Mathematical Institute,} Nagoya University 